\title{
FIELDS OF DEFINITION FOR DIVISION ALGEBRAS
}

\author{
M. LORENZ, Z. REICHSTEIN, L. H. ROWEN, AND D. J. SALTMAN
}

AbSTRACT. Let $A$ be a finite-dimensional division algebra containing a base field $k$ in its center $F$. We say that $A$ is defined over a subfield $F_{0}$ if there exists an $F_{0}$-algebra $A_{0}$ such that $A=A_{0} \otimes_{F_{0}} F$. We show that: (i) In many cases $A$ can be defined over a rational extension of $k$. (ii) If $A$ has odd degree $n \geq 5$, then $A$ is defined over a field $F_{0}$ of transcendence degree $\leq \frac{1}{2}(n-1)(n-2)$ over $k$. (iii) If $A$ is a $\mathbb{Z} / m \times \mathbb{Z} / 2$-crossed product for some $m \geq 2$ (and in particular, if $A$ is any algebra of degree 4 ) then $A$ is Brauer equivalent to a tensor product of two symbol algebras. Consequently, $\mathrm{M}_{m}(A)$ can be defined over a field $F_{0}$ such that $\operatorname{trdeg}_{k}\left(F_{0}\right) \leq 4$. (iv) If $A$ has degree 4 then the trace form of $A$ can be defined over a field $F_{0}$ of transcendence degree $\leq 4$. (In (i), (iii) and (iv) we assume that the center of $A$ contains certain roots of unity.)

\section{Contents}

1. Introduction 2

1.1. Parameter reduction 2

1.2. Rational fields of definition 3

1.3. $\mathbb{Z} / m \times \mathbb{Z} / 2$-crossed products 4

1.4. Fields of definition of quadratic forms 4

2. Preliminaries 5

2.1. G-lattices 5

2.2. Extension sequences 5

2.3. Twisted multiplicative $\mathcal{G}$-fields 6

2.4. Rational specialization 8

3. $\mathcal{G} / \mathcal{H}$-crossed products 9

4. Proof of Theorem 1.1 12

1991 Mathematics Subject Classification. 16K20, 16W22, 20C10, 20J06, 11E81.

Key words and phrases. division algebra, central simple algebra, crossed product, symbol algebra, cyclic algebra, biquaternion algebra, Brauer factor set, Brauer group, integral representation, $\mathcal{G}$-lattice, quadratic form, trace form, Pfister form, essential dimension.

M. Lorenz was supported in part by NSF grant DMS-9988756.

Z. Reichstein was supported in part by NSF grant DMS-9801675 and an NSERC research grant.

L. H. Rowen was supported by the Israel Science Foundation, founded by the Israel Academy of Sciences and Humanities - Center of Excellence Program no. 8007/99-3.

D. J. Saltman was supported in part by NSF grant DMS-9970213. 
$\begin{array}{ll}\text { 5. Proof of Theorem 1.2 } & 14\end{array}$

5.1. Proof of part (a) 14

5.2. Proof of part(b) 16

$\begin{array}{ll}\text { 6. Proof of Theorem } 1.3 & 17\end{array}$

7. The field of definition of a quadratic form 19

$\begin{array}{lr}\text { 7.1. Preliminaries } & 19\end{array}$

7.2. Proof of Theorem 1.5 21

$\begin{array}{ll}\text { References } & 22\end{array}$

\section{INTRODUCTION}

Let $F$ be a field and $A$ a finite-dimensional $F$-algebra. We say that $A$ is defined over a subfield $F_{0} \subset F$ if there exists an $F_{0}$-algebra $A_{0}$ such that $A=A_{0} \otimes_{F_{0}} F$.

Throughout this paper we will assume that $A$ is a finite-dimensional central simple $F$-algebra, and $F$ (and $F_{0}$ ) contain a base field $k$.

1.1. Parameter reduction. If $A$ is defined over $F_{0}$ and $\operatorname{trdeg}_{k}\left(F_{0}\right)<\operatorname{trdeg}_{k}(F)$ then the passage from $A$ to $A_{0}$ may be viewed as "parameter reduction" in $A$. This leads to the following natural question:

What is the smallest value of $\operatorname{trdeg}_{k}\left(F_{0}\right)$ such that $A$ is defined over $F_{0}$ ?

This number is clearly finite; we shall denote it by $\tau(A)$. Of particular interest to us will be the case where $A=\mathrm{UD}(n)$ is the universal division algebra of degree $n$ and $F=Z(n)$ is the center of $\operatorname{UD}(n)$. Recall that $\operatorname{UD}(n)$ is the subalgebra of $\mathrm{M}_{n}\left(k\left(x_{i j}, y_{i j}\right)\right)$ generated, as a $k$-division algebra, by two generic $n \times n$-matrices $X=\left(x_{i j}\right)$ and $Y=\left(y_{i j}\right)$, where $x_{i j}$ and $y_{i j}$ are $2 n^{2}$ independent commuting variables over $k$; see, e.g., $\mathrm{Pr}$, Section II.1], [Row 1 , Section 3.2] or $\mathrm{Sa}_{3}$, Section 14]. We shall write $d(n)$ for $\tau(\operatorname{UD}(n))$. It is easy to show that $d(n) \geq \tau(A)$ for any central simple algebra $A$ of degree $n$ whose center contains $k$; see Remark 2.8 (cf. also [Re $\mathrm{Re}_{2}$ Lemma 9.2]). In other words, every central simple algebra of degree $n$ can be "reduced to at most $d(n)$ parameters".

To the best of our knowledge, the earliest attempt to determine the value of $d(n)$ is due to Procesi, who showed that $d(n) \leq n^{2}$; see $[\mathrm{Pr}$, Thm. 2.1]. If $n=2,3$ or 6 and $k$ contains a primitive $n^{\text {th }}$ root of unity then $d(n)=2$, because $\mathrm{UD}(n)$ is cyclic for these $n$ and we can take $A_{0}$ to be a symbol algebra; cf. $\mathrm{Re}_{2}$, Lemma 9.4]. Rost Rost recently proved that

$$
d(4)=5
$$


For other $n$ the exact value of $d(n)$ is not known. However, the following inequalities hold:

$$
\begin{gathered}
d(n) \leq n^{2}-3 n+1 \quad \text { if } n \geq 4 \quad[\mathrm{Le}] \\
d(n) \leq d(n m) \leq d(n)+d(m) \quad \text { if }(n, m)=1 \quad\left[\mathrm{Re}_{2},\right. \text { Sect. 9.4], } \\
d\left(n^{r}\right) \geq 2 r \quad\left[\mathrm{Re}_{1},\right. \text { Theorem 16.1], } \\
d(n) \leq \frac{1}{2}(n-1)(n-2)+n \quad \text { for odd } n \quad \mathrm{Row}_{2} ; \text { cf. }\left[\mathrm{Re}_{2},\right. \text { Sect. 9.3]. }
\end{gathered}
$$

In this paper we will sharpen the last inequality by showing that

$$
d(n) \leq \frac{1}{2}(n-1)(n-2)
$$

for every odd $n \geq 5$. Moreover, in $\mathrm{UD}(n)$, reduction to this number of parameters can be arranged in a particularly nice fashion:

Theorem 1.1. Let $n \geq 5$ be an odd integer, $\operatorname{UD}(n)$ the universal division algebra of degree $n$, and $Z(n)$ its center. Then there exists a subfield $F$ of $Z(n)$ and a division algebra $D$ of degree $n$ with center $F$ such that

(a) $\mathrm{UD}(n)=D \otimes_{F} Z(n)$,

(b) $\operatorname{trdeg}_{k}(F)=\frac{1}{2}(n-1)(n-2)$, and

(c) $Z(n)$ is a rational extension of $F$.

In particular, $d(n) \leq \frac{1}{2}(n-1)(n-2)$.

We remark that in the language of $\mathrm{Re}_{2}$, the last assertion of Theorem 1.1 can be written as

$$
\operatorname{ed}\left(\mathrm{PGL}_{n}\right) \leq \frac{1}{2}(n-1)(n-2)
$$

1.2. Rational fields of definition. Another natural question is whether or not a given central simple algebra $A$ can be defined over a rational extension of $k$. We give the following partial answer to this question.

Theorem 1.2. Let $A$ be a finite-dimensional central simple algebra with center $F$ and let $t_{1}, t_{2}, \ldots$ be algebraically independent central indeterminates over $F$.

(a) Assume $\operatorname{deg}(A)=2^{i} p_{1} \ldots p_{r}$, where $i=0,1$ or 2 and $p_{1}, \ldots, p_{r}$ are distinct odd primes. Then for $s \gg 0$ the algebra $A\left(t_{1}, \ldots, t_{s}\right)$ is defined over a rational extension of $k$.

(b) Suppose the center of $A$ contains a primitive $e^{\text {th }}$ root of unity, where $e$ is the exponent of $A$. Then there exists an $r \geq 1$ such that for $s \gg 0$ the algebra $M_{r}(A)\left(t_{1}, \ldots, t_{s}\right)$ is defined over a rational extension of $k$. (Here we are imposing no restrictions on the degree of $A$.)

Here $A\left(t_{1}, \ldots, t_{s}\right)$ stands for $A \otimes_{F} F\left(t_{1}, \ldots, t_{s}\right)$ and similarly for $M_{r}(A)\left(t_{1}, \ldots, t_{s}\right)$. Note that part (b) may be interpreted as saying that for $s \gg 0$ the Brauer class of $A\left(t_{1}, \ldots, t_{s}\right)$ is defined over a rational extension of $k$. 
1.3. $\mathbb{Z} / m \times \mathbb{Z} / 2$-crossed products. As usual, we let $(a, b)_{m}$ denote the symbol algebra

$$
F\{x, y\} /\left(x^{m}=a, y^{m}=b, x y=\zeta_{m} y x\right),
$$

where $a, b \in F^{*}$ and $\zeta_{m}$ is a (fixed) primitive $m^{\text {th }}$ root of unity in $F$; cf. Row 3 , pp. 194-197]. In Section [6 we will prove the following:

Theorem 1.3. Let $A$ be a $\mathbb{Z} / m \times \mathbb{Z} / 2$-crossed product central simple algebra whose center $F$ contains a primitive $2 m^{\text {th }}$ root of unity $\zeta_{2 m}$. Then $A$ is Brauer equivalent (over $F)$ to $(a, b)_{m} \otimes_{F}(c, d)_{2 m}$ for some $a, b, c, d \in F^{*}$. (In other words, $M_{m}(A)$ is isomorphic to $(a, b)_{m} \otimes_{F}(c, d)_{2 m}$.)

Note that Theorem 1.3 applies to any division algebra of degree 4, since, by a theorem of Albert, any such algebra is a $\mathbb{Z} / 2 \times \mathbb{Z} / 2$-crossed product. In this setting our argument yields, in particular, an elementary proof of [S], Theorem 2, p. 288]. We also remark that Theorem 1.3 may be viewed as an explicit form of the Merkurjev-Suslin theorem for $\mathbb{Z} / m \times \mathbb{Z} / 2$-crossed products.

Letting $F_{0}=k\left(\zeta_{2 m}, a, b, c, d\right)$, we note that

$$
(a, b)_{m} \otimes_{F}(c, d)_{2 m}=\left((a, b)_{m} \otimes_{F_{0}}(c, d)_{2 m}\right) \otimes_{F_{0}} F
$$

Thus Theorem 1.3 shows that $\mathrm{M}_{m}(A)$ is defined over $F_{0}$. Since $\operatorname{trdeg}\left(F_{0}\right) \leq 4$, we obtain the following:

Corollary 1.4. Let $A$ be a $\mathbb{Z} / m \times \mathbb{Z} / 2$-crossed product central simple algebra whose center contains a primitive $2 m^{\text {th }}$ root of unity. Then $\tau\left(M_{m}(A)\right) \leq 4$. In particular, $\tau\left(M_{2}(A)\right) \leq 4$ for every central simple algebra $A$ of degree 4 whose center contains a primitive $4^{\text {th }}$ root of unity.

Note that the last assertion complements, in a somewhat surprising way, the above-mentioned result of Rost (1.1). Indeed, suppose the base field $k$ contains a primitive $4^{\text {th }}$ root of unity. Then for $A=\mathrm{UD}(4)$, Rost's theorem says that $\tau(A)=5$, where as Corollary 1.4 says that $\tau\left(\mathrm{M}_{2}(A)\right) \leq 4$.

1.4. Fields of definition of quadratic forms. A quadratic form $q: V \rightarrow F$ on an $F$-vector space $V=F^{n}$ is said to be defined over a subfield $F_{0}$ of $F$ if $q=q_{F_{0}} \otimes F$, where $q_{F_{0}}$ is a quadratic form on $V_{0}=F_{0}^{n}$.

In the last section we discuss fields of definition of quadratic forms. Of particular interest to us will be trace forms of central simple algebras of degree 4 , recently studied by Rost, Serre and Tignol [RST. (Recall that the trace form of a central simple algebra $A$ is the quadratic form $x \mapsto \operatorname{Tr}\left(x^{2}\right)$ defined over the center of $A$.) We will use a theorem of Serre [Se] (see our Proposition [7.3) to prove the following:

Theorem 1.5. Let $A$ be a central simple algebra of degree 4 whose center $F$ contains a primitive $4^{\text {th }}$ root of unity. Then the trace form of $A$ is defined over a subfield $F_{0} \subset F$ such that $\operatorname{trdeg}_{k}\left(F_{0}\right) \leq 4$. 
Note that Theorem 1.5 may also be viewed as complementing (1.1).

Acknowledgment. The authors would like to thank the referee for a number of helpful and constructive comments and for catching several mistakes in an earlier version of this paper.

\section{Preliminaries}

2.1. $\mathcal{G}$-lattices. Throughout, $\mathcal{G}$ will denote a finite group and $\mathcal{H}$ will be a subgroup of $\mathcal{G}$. Recall that a $\mathcal{G}$-module is a (left) module over the integral group

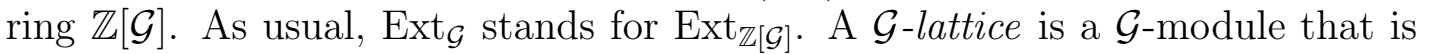
free of finite rank over $\mathbb{Z}$. Further, a $\mathcal{G}$-lattice $M$ is called

- a permutation lattice if $M$ has a $\mathbb{Z}$-basis that is permuted by $\mathcal{G}$;

- permutation projective (or invertible) if $M$ is a direct summand of some permutation $\mathcal{G}$-lattice.

A $\mathcal{G}$-module $M$ is called faithful if no $1 \neq g \in \mathcal{G}$ acts as the identity on $M$.

The $\mathcal{G} / \mathcal{H}$-augmentation kernel is defined as the kernel of the natural augmentation map

$$
\mathbb{Z}[\mathcal{G} / \mathcal{H}]=\mathbb{Z}[\mathcal{G}] \otimes_{\mathbb{Z}[\mathcal{H}]} \mathbb{Z} \longrightarrow \mathbb{Z}, \quad \bar{g}=g \otimes 1 \mapsto 1 \quad(g \in \mathcal{G}) .
$$

Thus there is a short exact sequence of $\mathcal{G}$-lattices

$$
0 \rightarrow \omega(\mathcal{G} / \mathcal{H}) \rightarrow \mathbb{Z}[\mathcal{G} / \mathcal{H}] \rightarrow \mathbb{Z} \rightarrow 0
$$

$\omega(\mathcal{G} /\{1\})$ will be written as $\omega \mathcal{G}$; this is the ordinary augmentation ideal of the group ring $\mathbb{Z}[\mathcal{G}]$; cf. $[\mathrm{Pa}$, Chap. 3].

2.2. Extension sequences. Exact sequences of $\mathcal{G}$-lattices of the form

$$
\begin{aligned}
& 0 \rightarrow M \rightarrow P \rightarrow \omega(\mathcal{G} / \mathcal{H}) \rightarrow 0, \\
& \text { with } P \text { permutation and } M \text { faithful }
\end{aligned}
$$

will play an important role in the sequel. In this subsection we introduce two such sequences, (2.3) and (2.4).

Let $d_{\mathcal{G}}(\omega(\mathcal{G} / \mathcal{H}))$ denote the minimum number of generators of $\omega(\mathcal{G} / \mathcal{H})$ as a $\mathbb{Z}[\mathcal{G}]$-module. Then for any $r \geq d_{\mathcal{G}}(\omega(\mathcal{G} / \mathcal{H}))$ there exists an exact sequence

$$
0 \rightarrow M \rightarrow \mathbb{Z}[\mathcal{G}]^{r} \stackrel{f}{\rightarrow} \omega(\mathcal{G} / \mathcal{H}) \rightarrow 0
$$

of $\mathcal{G}$-lattices.

Lemma 2.1. $M$ is a faithful $\mathcal{G}$-lattice if and only if $r \geq 2$ or $\mathcal{H} \neq\{1\}$.

Proof. It is enough to show that $M \otimes \mathbb{Q}$ is $\mathcal{G}$-faithful; thus we may work over the semisimple algebra $\mathbb{Q}[\mathcal{G}]$. Since $f \otimes \mathbb{Q}$ splits, we have a $\mathbb{Q}[\mathcal{G}]$-isomorphism $(\omega(\mathcal{G} / \mathcal{H}) \otimes \mathbb{Q}) \oplus(M \otimes \mathbb{Q}) \simeq \mathbb{Q}[\mathcal{G}]^{r}$. Similarly, the canonical exact sequence 
$\mathbb{Z}[\mathcal{G}] \omega \mathcal{H} \longmapsto \mathbb{Z}[\mathcal{G}] \rightarrow \mathbb{Z}[\mathcal{G} / \mathcal{H}]$ gives $(\omega(\mathcal{G} / \mathcal{H}) \otimes \mathbb{Q}) \oplus \mathbb{Q} \oplus \mathbb{Q}[\mathcal{G}] \omega \mathcal{H} \simeq \mathbb{Q}[\mathcal{G}]$. Therefore,

$$
M \otimes \mathbb{Q} \simeq \mathbb{Q}[\mathcal{G}]^{r-1} \oplus \mathbb{Q} \oplus \mathbb{Q}[\mathcal{G}] \omega \mathcal{H}
$$

If $r \geq 2$ then $\mathbb{Q}[\mathcal{G}]^{r-1}$ is $\mathcal{G}$-faithful, and if $\mathcal{H} \neq\{1\}$ then $\omega \mathcal{H} \otimes \mathbb{Q}$ is $\mathcal{H}$-faithful and so $\mathbb{Q}[\mathcal{G}] \omega \mathcal{H} \simeq(\omega \mathcal{H} \otimes \mathbb{Q}) \uparrow_{\mathcal{H}}^{\mathcal{G}}$ is $\mathcal{G}$-faithful. In either case, $M \otimes \mathbb{Q}$ is faithful, as desired. On the other hand, $r=1$ and $\mathcal{H}=\{1\}$ leads to $M \otimes \mathbb{Q} \simeq \mathbb{Q}$ which is not faithful.

Lemma 2.2. There is an exact sequence

$$
0 \rightarrow \omega(\mathcal{G} / \mathcal{H})^{\otimes 2} \stackrel{m}{\rightarrow} P \rightarrow \omega(\mathcal{G} / \mathcal{H}) \rightarrow 0
$$

where $P$ is the (permutation) sublattice $P=\bigoplus_{\overline{g_{1}} \neq \overline{g_{2}} \in \mathcal{G} / \mathcal{H}} \mathbb{Z}\left(\overline{g_{1}} \otimes \overline{g_{2}}\right)$ of $\mathbb{Z}[\mathcal{G} / \mathcal{H}]^{\otimes 2}$. The lattice $\omega(\mathcal{G} / \mathcal{H})^{\otimes 2}$ is faithful if and only if $\mathcal{H}$ contains no normal subgroup $\neq\{1\}$ of $\mathcal{G}$ and $[\mathcal{G}: \mathcal{H}] \geq 3$.

Proof. Tensoring sequence (2.1) with $\omega(\mathcal{G} / \mathcal{H})$ and putting $P^{\prime}=\omega(\mathcal{G} / \mathcal{H}) \otimes$ $\mathbb{Z}[\mathcal{G} / \mathcal{H}]$, we obtain an exact sequence

$$
0 \rightarrow \omega(\mathcal{G} / \mathcal{H})^{\otimes 2} \rightarrow P^{\prime} \rightarrow \omega(\mathcal{G} / \mathcal{H}) \rightarrow 0
$$

where $\otimes=\otimes_{\mathbb{Z}}$. The elements $\left(\overline{g_{1}}-\overline{g_{2}}\right) \otimes \overline{g_{2}}$ with $\overline{g_{1}} \neq \overline{g_{2}} \in \mathcal{G} / \mathcal{H}$ form a $\mathbb{Z}$-basis of $P^{\prime}$, and the map

$$
m:\left(\overline{g_{1}}-\overline{g_{2}}\right) \otimes \overline{g_{2}} \mapsto \overline{g_{1}} \otimes \overline{g_{2}}
$$

is a $\mathcal{G}$-isomorphism $P^{\prime} \simeq P$.

For the faithfulness assertion, note that $\mathcal{N}=\bigcap_{g \in \mathcal{G}} \mathcal{H}^{g}$ acts trivially on $\mathbb{Z}[\mathcal{G} / \mathcal{H}]$ and hence on $\omega(\mathcal{G} / \mathcal{H})^{\otimes 2}$; so $\mathcal{N}=\{1\}$ is surely required for faithfulness. Also, if $[\mathcal{G}: \mathcal{H}] \leq 2$ then $\mathcal{G}$ acts trivially on $\omega(\mathcal{G} / \mathcal{H})^{\otimes 2}$. Conversely, if $\mathcal{N}=\{1\}$ and $[\mathcal{G}: \mathcal{H}] \geq 3$ then it is easy to verify that $\omega(\mathcal{G} / \mathcal{H})^{\otimes 2}$ is indeed faithful.

2.3. Twisted multiplicative $\mathcal{G}$-fields. Recall that a $\mathcal{G}$-field is a field $F$ on which the finite group $\mathcal{G}$ acts by automorphisms, written $f \mapsto g(f)$. Morphisms of $\mathcal{G}$-fields are $\mathcal{G}$-equivariant field homomorphisms. The $\mathcal{G}$-field $F$ is called faithful if every $1 \neq g \in \mathcal{G}$ acts non-trivially on $F$. If $K \subseteq F$ is a field extension and $V \subseteq F$ a subset of $F$ (not necessarily algebraically independent over $K$ ) then we let $K(V)$ denote the subfield of $F$ that is generated by $K$ and $V$.

Lemma 2.3. (cf. Sh Appendix 3]) Let $K \subseteq F$ be an extension of $\mathcal{G}$-fields with $K$ faithful. Assume that $F=K(V)$ for some $\mathcal{G}$-stable $K$-subspace $V \subseteq F$. Then

(a) $V=K V^{\mathcal{G}}$, where $V^{\mathcal{G}}$ denotes the $\mathcal{G}$-invariants in $V$,

(b) $F=K\left(V^{\mathcal{G}}\right)$, and

(c) $F^{\mathcal{G}}=K^{\mathcal{G}}\left(V^{\mathcal{G}}\right)$.

Proof. (a) Let $S=K \# \mathcal{G}$ denote the skew group ring for the given $\mathcal{G}$-action on $K$. The $\mathcal{G}$-action on $F$ and multiplication with $K$ make $F$ a (left) $S$-module, and $V$ is a submodule. Moreover, since $K$ is a faithful $\mathcal{G}$-field, $S$ is a simple ring; 
see, e.g., [J, p. 473]. In particular, the element $t=\sum_{g \in \mathcal{G}} g \in S$ generates $S$ as a 2-sided ideal. Thus, $S=S t S=K t K$ and consequently, $V=K t K V=K V^{\mathcal{G}}$.

(b) is an immediate consequence of (a).

(c) Let $E=K^{\mathcal{G}}\left(V^{\mathcal{G}}\right)$. We want to show that $E=F^{\mathcal{G}}$. Clearly $E \subseteq F^{\mathcal{G}}$. To prove equality, note that $K E$ is a subring of $F$ containing $K$ and $V^{\mathcal{G}}$, and that $\operatorname{dim}_{E} K E \leq \operatorname{dim}_{K^{\mathcal{G}}} K=|\mathcal{G}|$. Thus, $K E$ is a field, and hence (b) implies that $K E=F$. Therefore, $\operatorname{dim}_{E} F \leq|\mathcal{G}|=\operatorname{dim}_{F^{\mathcal{G}}} F$. Since $E \subseteq F^{\mathcal{G}}$, this is only possible if $E=F^{\mathcal{G}}$.

We recall a well-known construction of $\mathcal{G}$-fields; cf. [Sa . Given a $\mathcal{G}$-field $E$, a $\mathcal{G}$-lattice $M$, and an extension class $\gamma \in \operatorname{Ext}_{\mathcal{G}}\left(M, E^{*}\right)$, the twisted multiplicative $\mathcal{G}$-field $E_{\gamma}(M)$ is constructed as follows. Form the group algebra $E[M]$ of $M$ over $E$; this is a commutative integral domain with group of units $\mathrm{U}(E[M])=E^{*} \times M$. We shall use multiplicative notation for $M$ in this setting. Let $E(M)$ denote the field of fractions of $E[M]$. Choose an extension of $\mathcal{G}$-modules

$$
1 \rightarrow E^{*} \rightarrow V \rightarrow M \rightarrow 1
$$

representing $\gamma$. So, as abelian groups, $V \simeq \mathrm{U}(E[M])$. Using this identification, we obtain a $\mathcal{G}$-action on $\mathrm{U}(E[M])$ inducing the given action on $E^{*}$. The action of $\mathcal{G}$ on $\mathrm{U}(E[M])$ extends uniquely to $E[M]$, and to $E(M)$; we will use $E_{\gamma}[M]$ and $E_{\gamma}(M)$ to denote $E[M]$ and $E(M)$ with the $\mathcal{G}$-actions thus obtained. For $\gamma=1$, we will simply write $E[M]$ and $E(M)$. We remark that the choice of the sequence (2.5) representing a given $\gamma \in \operatorname{Ext}_{\mathcal{G}}\left(M, E^{*}\right)$ is insubstantial: a different choice leads to $\mathcal{G}$-isomorphic results.

For future reference, we record the following application of Lemma 2.3 essentially due to Masuda [Mas]; see also [Le, Proposition 1.6], [Sa, Lemma 12.8].

Proposition 2.4. Let $E$ be a faithful $\mathcal{G}$-field and let $P$ be a permutation $\mathcal{G}$-lattice. Then any twisted multiplicative $\mathcal{G}$-field $E_{\gamma}(P)$ can be written as

$$
E_{\gamma}(P)=E\left(t_{1}, \ldots, t_{n}\right)
$$

with $\mathcal{G}$-invariant transcendental (over $E$ ) elements $t_{i}$. In particular, $E_{\gamma}(P)^{\mathcal{G}}=$ $E^{\mathcal{G}}\left(t_{1}, \ldots, t_{n}\right)$ is rational over $E^{\mathcal{G}}$.

Proof. We have an extension of $\mathcal{G}$-modules $1 \rightarrow E^{*} \rightarrow \mathrm{U}\left(E_{\gamma}[P]\right) \rightarrow P \rightarrow 1$ representing $\gamma$, as in (2.5). Fix a $\mathbb{Z}$-basis, $X$, of $P$ that is permuted by the action of $\mathcal{G}$. For each $x \in X$, choose a preimage $x^{\prime} \in \mathrm{U}\left(E_{\gamma}[P]\right)$. Then $\left\{x^{\prime}\right\}_{x \in X}$ is a collection of transcendental generators of $E_{\gamma}(P)$ over $E$, and $\mathcal{G}$ acts via $g\left(x^{\prime}\right)=g(x)^{\prime} y$ for some $y=y(g, x) \in E^{*}$. Letting $V$ denote the $E$-subspace of $E_{\gamma}(P)$ that is generated by $\left\{x^{\prime}\right\}_{x \in X}$, we conclude from Lemma 2.3 that $V$ has a basis consisting of $\mathcal{G}$-invariant elements, say $t_{1}, \ldots, t_{n}$, and $E_{\gamma}(P)=$ $E\left(t_{1}, \ldots, t_{n}\right), E_{\gamma}(P)^{\mathcal{G}}=E^{\mathcal{G}}\left(t_{1}, \ldots, t_{n}\right)$. The $t_{i}$ are transcendental over $E$, since $\operatorname{trdeg}_{E} E_{\gamma}(P)=\operatorname{rank}(P)=n$. 
2.4. Rational specialization. Let $A / F$ and $B / K$ be central simple algebras. We will call $B / K$ a rational specialization of $A / F$ if there exists a field $F^{\prime}$ containing both $F$ and $K$ such that $F^{\prime} / K$ is rational and

$$
B \otimes_{K} F^{\prime} \simeq A \otimes_{F} F^{\prime} .
$$

In other words, $B$ is a rational specialization of $A$ if $\operatorname{deg} A=\operatorname{deg} B$ and $A$ embeds in some $B\left(t_{1}, \ldots, t_{n}\right)$, where $t_{1}, t_{2}, \ldots$ are independent variables over $F$; cf. [Sa p. 73],

For the rest of this paper we fix an (arbitrary) base field $k$. All other fields are understood to contain a copy $k$ and all maps (i.e., inclusions) between fields are understood to restrict to the identity map on $k$.

Definition 2.5. Let $\Lambda$ be a class of central simple algebras. We shall say that an algebra $A \in \Lambda$ has the rational specialization property in the class $\Lambda$ if every $B \in \Lambda$ is a rational specialization of $A$. If $\Lambda$ is the class of all central simple algebras of degree $n=\operatorname{deg}(A)$ then we will omit the reference to $\Lambda$ and will simply say that $A$ has the rational specialization property.

Example 2.6. By [RV] Lemma 3.1], UD $(n)$ has the rational specialization property. This is also implicit in $\mathrm{Sa}_{2}$. We remark that any central simple algebra $A / F$ with the rational specialization property is a division algebra. To see this, specialize $A$ to $\operatorname{UD}(n)$, where $n=\operatorname{deg}(A)$.

Recall the definition of $\tau(A)$ given at the beginning of this paper.

Lemma 2.7. Let $A / F$ and $B / K$ be a central simple algebras.

(a) If $A^{\prime} \simeq A \otimes_{F} F^{\prime}$ for some rational field extension $F^{\prime} / F$ then $\tau(A)=\tau\left(A^{\prime}\right)$.

(b) (cf. $\mathrm{Sa}_{3}$, Lemma 11.1]) If $A$ is a rational specialization of $B$ then $\tau(A) \leq$ $\tau(B)$.

Proof. (a) The inequality $\tau\left(A^{\prime}\right) \leq \tau(A)$ is immediate from the definition of $\tau$. To prove the opposite inequality, suppose $A^{\prime} \simeq A_{0} \otimes_{F_{0}} F^{\prime}$, where $A_{0}$ is a central simple algebra over an intermediate field $k \subset F_{0} \subset F^{\prime}$, and $A_{0}$ is chosen so that $\operatorname{trdeg}_{k}\left(F_{0}\right)=\tau\left(A^{\prime}\right)$. In particular, $\operatorname{trdeg}_{k}\left(F_{0}\right) \leq \operatorname{trdeg}_{k}(F)$. Then by $\mathrm{RV}$, Proposition 3.2], $A_{0}$ embeds in $A$, i.e., $A \simeq A_{0} \otimes_{F_{0}} F$ for some embedding $F_{0} \hookrightarrow F$. Consequently, $\tau(A) \leq \operatorname{trdeg}_{k}(K)=\tau\left(A^{\prime}\right)$, as desired.

(b) We may assume $B \otimes_{K} F^{\prime}=A^{\prime}$, as in (a). Clearly $\tau(B) \geq \tau\left(A^{\prime}\right)$, and part (a) tells us that $\tau\left(A^{\prime}\right)=\tau(A)$.

Remark 2.8. Combining Example 2.6 with Lemma 2.7(b), we see that $\tau(A) \leq$ $d(n)=\tau(\mathrm{UD}(n))$ holds for every central simple algebra $A$ of degree $n$; cf. $\mathrm{Re}_{2}$, Lemma 9.2]. 


\section{3. $\mathcal{G} / \mathcal{H}$-CROSSED PRODUCTS}

We shall call a central simple algebra $A / F$ an $(E, \mathcal{G} / \mathcal{H})$-crossed product if $A$ has a maximal subfield $L$ whose Galois closure $E$ over $F$ has the property that $\operatorname{Gal}(E / F)=\mathcal{G}$ and $\operatorname{Gal}(E / L)=\mathcal{H}$. (We adopt the convention that a maximal subfield of $A$ is a subfield $L$ that is maximal as a commutative subring; so $[L: F]$ is equal to the degree of $A$.) We will say that $A$ is a $\mathcal{G} / \mathcal{H}$-crossed product if it is an $(E, \mathcal{G} / \mathcal{H})$-crossed product for some faithful $\mathcal{G}$-field $E$. If $\mathcal{H}=\{1\}$ then a $\mathcal{G} / \mathcal{H}$-crossed product is just a $\mathcal{G}$-crossed product in the usual sense (see, e.g., Row $_{1}$, Definition 3.1.23]).

Example 3.1. Consider the universal division algebra $\mathrm{UD}(n)$ generated by two generic matrices, $X$ and $Y$, over $k$. Denote the center of this algebra by $Z(n)$. Setting $L=Z(n)(X)$, we see that $\mathrm{UD}(n)$ is an $\mathcal{S}_{n} / \mathcal{S}_{n-1}$-crossed product $\mathrm{Pr}$, Theorem 1.9]; see also Section 4 below.

Since the degree of a $\mathcal{G} / \mathcal{H}$-crossed product is equal to $[\mathcal{G}: \mathcal{H}]$, we see that isomorphism classes of $(E, \mathcal{G} / \mathcal{H})$-crossed products are in 1-1 correspondence with the relative Brauer group $\mathbf{B}(L / F)$, which, in turn, is naturally identified with the kernel of the restriction homomorphism $H^{2}\left(\mathcal{G}, E^{*}\right) \rightarrow H^{2}\left(\mathcal{H}, E^{*}\right)$; cf. [Pi, 14.7].

A $\mathcal{G}$-module $M$ is called $H^{1}$-trivial if $H^{1}(\mathcal{H}, M)=0$ holds for every subgroup $\mathcal{H} \leq \mathcal{G}$. Equivalently, $M$ is $H^{1}$-trivial if $\operatorname{Ext}_{\mathcal{G}}(P, M)=0$ for all permutation projective $\mathcal{G}$-lattices $P$; see, e.g., $\mathrm{Sa}_{3}$, Lemma 12.3].

Lemma 3.2. Given an exact sequence

$$
0 \rightarrow M \rightarrow P \rightarrow \omega(\mathcal{G} / \mathcal{H}) \rightarrow 0,
$$

of $\mathcal{G}$-lattices, with $P$-permutation, let $N$ be an $H^{1}$-trivial $\mathcal{G}$-module. Denote the kernel of the restriction homomorphism $H^{2}(\mathcal{G}, N) \rightarrow H^{2}(\mathcal{H}, N)$ by $K(\mathcal{G} / \mathcal{H}, N)$. Then there is a natural isomorphism

$$
\phi_{N}: \operatorname{Hom}_{\mathcal{G}}(M, N) / \operatorname{Im}\left(\operatorname{Hom}_{\mathcal{G}}(P, N)\right) \stackrel{\simeq}{\longrightarrow} K(\mathcal{G} / \mathcal{H}, N) .
$$

Here "natural" means that for every homomorphism $N \rightarrow N^{\prime}$ of $H^{1}$-trivial $\mathcal{G}$-modules, the following diagram commutes

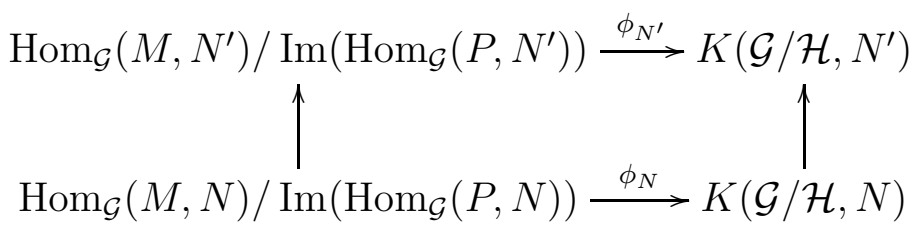

Note that, other than in sequence (2.2), the $\mathcal{G}$-lattice $M$ need not be faithful.

Proof. The lemma is a variant of $\left[\mathrm{Sa}_{3}\right.$, Theorem 12.10], where the same assertion is made for the sequence (2.4). The proof of [Sa, Theorem 12.10] goes through unchanged in our setting. 
In subsequent applications we will always take $N=E^{*}$, where $E$ is a faithful $\mathcal{G}$-field. Note that $E^{*}$ is $H^{1}$-trivial by Hilbert's Theorem 90 . As we remarked above, $K\left(\mathcal{G} / \mathcal{H}, E^{*}\right)$ is naturally identified with $\mathbf{B}(L / F)$, where $L=E^{\mathcal{H}}$, and elements of $\mathbf{B}(L / F)$ are in 1-1 correspondence with $(E, \mathcal{G} / \mathcal{H})$-crossed products. We shall denote the $(E, \mathcal{G} / \mathcal{H})$-crossed product associated to a $\mathcal{G}$-homomorphism $f: M \rightarrow E^{*}$ by $\operatorname{Alg}(f)$.

Lemma 3.3. Consider a sequence of $\mathcal{G}$-lattices of the form (2.2). Let $E$ be $\mathcal{G}$ field and $f: M \rightarrow E^{*}$ be a homomorphism of $\mathcal{G}$-modules. If $k(f(M))$ is contained in a faithful $\mathcal{G}$-subfield $E_{0}$ of $E$ then $\operatorname{Alg}(f)$ is defined over $E_{0}^{\mathcal{G}}$.

Proof. Since $f$ is the composition of $f_{0}: M \rightarrow E_{0}^{*}$ with the inclusion $E_{0}^{*} \hookrightarrow E^{*}$, Lemma 3.2 tells us that $A=\operatorname{Alg}\left(f_{0}\right) \otimes_{E_{0}^{\mathcal{G}}} E^{\mathcal{G}}$.

Remark 3.4. In the special case where the sequence

$$
0 \rightarrow M \rightarrow P \rightarrow \omega(\mathcal{G} / \mathcal{H}) \rightarrow 0
$$

is given by (2.4), $M=\omega(\mathcal{G} / \mathcal{H})^{\otimes 2}$ has a particularly convenient set of generators

$$
y_{i j h}=\left(\overline{g_{i}}-\overline{g_{j}}\right) \otimes\left(\overline{g_{j}}-\overline{g_{h}}\right),
$$

where $\mathcal{G} / \mathcal{H}=\left\{\overline{g_{1}}, \ldots, \overline{g_{n}}\right\}$ is the set of left cosets of $\mathcal{H}$ in $\mathcal{G}$ and $i, j, h$ range from 1 to $n=[\mathcal{G}: \mathcal{H}]$; cf. [RS, Lemma 1.2]. If $f: \omega(\mathcal{G} / \mathcal{H})^{\otimes 2} \rightarrow E^{*}$ is a $\mathcal{G}$-module homomorphism then the elements $c_{i j h}=f\left(y_{i j h}\right)$ form a reduced Brauer factor set for $\operatorname{Alg}(f)$ in the sense of [RS, p. 449]. Conversely, for any reduced Brauer factor set $\left(c_{i j h}\right)$ in $E^{*}$, there exists a homomorphism $f: \omega(\mathcal{G} / \mathcal{H})^{\otimes 2} \rightarrow E^{*}$ such that $f\left(y_{i j h}\right)=c_{i j h}$; see [RS, Corollary 1.3]. Thus Lemma 3.3 takes the following form:

Let $A$ be an $(E, \mathcal{G} / \mathcal{H})$-crossed product defined by a reduced Brauer factor set $\left(c_{i j h}\right)$. Suppose $\left(c_{i j h}\right)$ is contained in a faithful $\mathcal{G}$-subfield $E_{0}$ of $E$. Then $A$ is defined over $E_{0}^{\mathcal{G}}$.

This following theorem is a variant of $\mathrm{Sa}_{3}$, Theorem 12.11].

Theorem 3.5. Given the sequence (2.2), let $\mu: M \hookrightarrow k(M)^{*}$ be the natural inclusion. Then $D=\operatorname{Alg}(\mu)$ has the rational specialization property in the class of $\mathcal{G} / \mathcal{H}$-crossed products containing a copy of $k$ in their center. In particular, $\tau(A) \leq \operatorname{rank}(M)$ holds for any $\mathcal{G} / \mathcal{H}$-crossed product $A / F$ with $k \subset F$.

Proof. Write $A=\operatorname{Alg}(f)$ for some $\mathcal{G}$-homomorphism $f: M \rightarrow E^{*}$, where $E$ is a faithful $\mathcal{G}$-field with $E^{\mathcal{G}}=F$; see the remarks following Lemma 3.2 . Furthermore, let $E(P)$ denote the fraction field of the group algebra $E[P]$, with the $\mathcal{G}$-action induced from the $\mathcal{G}$-actions on $E$ and $P$. By Proposition 2.4 , there exists an $E$ isomorphism $j: E(P) \simeq E(\mathbf{t})$ of $\mathcal{G}$-fields, where $\mathbf{t}=\left(t_{1}, \ldots, t_{r}\right), r=\operatorname{rank}(P)$, are indeterminates on which $\mathcal{G}$ acts trivially. Therefore, $E(P)^{\mathcal{G}} \simeq E^{\mathcal{G}}(\mathbf{t})=F(\mathbf{t})$ is a rational extension of $F$. Let $f_{\mathbf{t}}: M \rightarrow E(\mathbf{t})^{*}$ denote the composition of $f$ with the 
natural inclusion $E^{*} \hookrightarrow E(\mathbf{t})^{*}$. Then $\operatorname{Alg}\left(f_{\mathbf{t}}\right)=\operatorname{Alg}(f) \otimes_{F} F(\mathbf{t})=A \otimes_{F} F(\mathbf{t})$. By Lemma 3.2. $\operatorname{Alg}\left(f_{\mathbf{t}}\right) \simeq \operatorname{Alg}\left(f_{\mathbf{t}}+\left.g\right|_{M}\right)$ for any $g \in \operatorname{Hom}_{\mathcal{G}}\left(P, E(\mathbf{t})^{*}\right)$. Let $g$ be the composite $g: P \hookrightarrow E(P)^{*} \stackrel{\sim}{\longrightarrow} E(\mathbf{t})^{*}$ and let $\varphi$ be the $\mathcal{G}$-module map $\varphi: M \rightarrow E(\mathbf{t})^{*}, \varphi(m)=f_{\mathbf{t}}(m) g(m)$. We claim that $\varphi$ lifts to an embedding of $\mathcal{G}$-fields $k(M) \hookrightarrow E(\mathbf{t})$. Indeed, modulo $E^{*}, \varphi(m) \equiv g(m) \in P \subseteq E(\mathbf{t})^{*}$. Hence, $\{\varphi(m)\}_{m \in M}$ is an $E$-linearly independent subset of $E(\mathbf{t})$, and so the map $k[\varphi]: k[M] \rightarrow E(\mathbf{t}), \sum_{m} k_{m} m \mapsto \sum_{m} k_{m} \varphi(m)$ is a $\mathcal{G}$-equivariant embedding of the group ring $k[M]$ into $E(\mathbf{t})$. This embedding lifts to an embedding of $\mathcal{G}$-fields $\phi: k(M)=Q(k[M]) \hookrightarrow E(\mathbf{t})$, as we have claimed. So $\phi \circ \mu=\varphi$, and hence $D \otimes_{k(M)^{\mathcal{G}}} F(\mathbf{t})=\operatorname{Alg}(\phi \circ \mu)=\operatorname{Alg}(\varphi) \simeq \operatorname{Alg}\left(f_{\mathbf{t}}\right)=A \otimes_{F} F(\mathbf{t})$. This proves that $A$ is a rational specialization of $D$.

Lemmas 2.7(b) and 3.3 now imply that $\tau(A) \leq \tau(D) \leq \operatorname{trdeg}_{k} k(M)^{\mathcal{G}}=$ $\operatorname{rank}(M)$. This completes the proof of the theorem.

Remark 3.6. Continuing with the notation used in the above theorem, the rational specialization property of $D=\operatorname{Alg}(\mu)$ implies that $D$ is a division algebra of exponent $[\mathcal{G}: \mathcal{H}]$. Indeed, by [FSS, Appendix] there exists a $\mathcal{G} / \mathcal{H}$ crossed product division algebra of exponent $[\mathcal{G}: \mathcal{H}]$, and the above assertion can be proved by specializing $D$ to this algebra. Alternatively, the fact that $D$ is a division algebra of exponent $[\mathcal{G}: \mathcal{H}]$ can be checked directly by showing that the image of $\mu$ in $H^{2}\left(\mathcal{G}, k(M)^{*}\right)$ (see Lemma 3.2) has order $[\mathcal{G}: \mathcal{H}]$.

Remark 3.7. The above construction applies in particular to sequences of the form (2.3). The following special type of sequence (2.3) has been particularly well-explored. Write $\mathcal{G}=\left\langle\mathcal{H}, g_{1}, \ldots, g_{r}\right\rangle$ for suitable $g_{i} \in \mathcal{G}$; the minimal such $r$ is usually denoted by $d(\mathcal{G} / \mathcal{H})$. Then we can define an epimorphism of $\mathcal{G}$ lattices $f: \mathbb{Z}[\mathcal{G}]^{r} \rightarrow \omega(\mathcal{G} / \mathcal{H}), f\left(\alpha_{1}, \ldots, \alpha_{r}\right)=\sum_{i=1}^{r} \alpha_{i} \overline{\left(g_{i}-1\right)}$, where $: \mathbb{Z}[\mathcal{G}] \rightarrow$ $\mathbb{Z}[\mathcal{G} / \mathcal{H}]$ is the canonical map; see $[\mathrm{Pa}$, Lemma 3.1.1]. The kernel $R(\mathcal{G} / \mathcal{H})=$ Ker $f$ is called a relative relation module; it has the following group theoretical description. Let $\mathcal{F}_{r}$ denote the free group on $r$ generators and consider the presentation

$$
1 \rightarrow \mathcal{R} \rightarrow \mathcal{F}_{r} * \mathcal{H} \rightarrow \mathcal{G} \rightarrow 1
$$

where $\mathcal{F}_{r} * \mathcal{H} \rightarrow \mathcal{G}$ is the identity on $\mathcal{H}$ and sends the $r$ generators of $\mathcal{F}_{r}$ to the elements $g_{1}, \ldots, g_{r}$. Then $R(\mathcal{G} / \mathcal{H}) \simeq \mathcal{R}^{\mathrm{ab}}=\mathcal{R} /[\mathcal{R}, \mathcal{R}]$, with $\mathcal{G}$ acting by conjugation; see $\mathrm{Ki}$ ] and $[\mathrm{Gr}$ (for $\mathcal{H}=\{1\}$ ). Thus, we have the following version of sequence (2.3) with $M=\mathcal{R}^{\mathrm{ab}}$ :

$$
0 \rightarrow \mathcal{R}^{\mathrm{ab}} \rightarrow \mathbb{Z}[\mathcal{G}]^{r} \rightarrow \omega(\mathcal{G} / \mathcal{H}) \rightarrow 0
$$

When $\mathcal{H}=\{1\}$ and $r \geq 2$, the division algebra $D$ constructed via (3.3) in Theorem 3.5 is identical with the generic $\mathcal{G}$-crossed product of Snider [S]; see also Rosset [Ro]. Explicitly:

Given a free presentation $1 \rightarrow \mathcal{R} \rightarrow \mathcal{F}_{r} \rightarrow \mathcal{G} \rightarrow 1$ of $\mathcal{G}$ with $r \geq 2$, let $M=\mathcal{R}^{\mathrm{ab}} \leq \overline{\mathcal{F}_{r}}=\mathcal{F}_{r} /[\mathcal{R}, \mathcal{R}]$ and let $a \in H^{2}\left(\mathcal{G}, k(M)^{*}\right)$ be the 
image of the extension class $\left[1 \rightarrow M \rightarrow \overline{\mathcal{F}_{r}} \rightarrow \mathcal{G} \rightarrow 1\right] \in H^{2}(\mathcal{G}, M)$ under the natural inclusion $\mu: M \hookrightarrow k(M)^{*}$. Then $D=\operatorname{Alg}(\mu)$ is the $\mathcal{G}$-crossed product $(k(M), \mathcal{G}, a)$ or, equivalently, the localization of the group algebra $k\left[\overline{\mathcal{F}_{r}}\right]$ at the nonzero elements of $k[M]$.

Corollary 3.8. Let $A$ be a $\mathcal{G} / \mathcal{H}$-crossed product and let $d_{\mathcal{G}}(\omega(\mathcal{G} / \mathcal{H}))$ be the minimal number of generators of $\omega(\mathcal{G} / \mathcal{H})$ as a $\mathcal{G}$-module. Then

$$
\tau(A) \leq r|\mathcal{G}|-[\mathcal{G}: \mathcal{H}]+1
$$

where

$$
r= \begin{cases}d_{\mathcal{G}}(\omega(\mathcal{G} / \mathcal{H})) & \text { if } \mathcal{H} \neq\{1\} \\ \max \left\{2, d_{\mathcal{G}}(\omega(\mathcal{G} / \mathcal{H}))\right\} & \text { if } \mathcal{H}=\{1\}\end{cases}
$$

Proof. Applying Theorem 3.5 to the exact sequence (2.3), we obtain

$$
\tau(A) \leq \operatorname{rank}(M)=\operatorname{rank}\left(\mathbb{Z}[G]^{r}\right)-\operatorname{rank}(\omega(\mathcal{G} / \mathcal{H}))=r|\mathcal{G}|-[\mathcal{G}: \mathcal{H}]+1,
$$

as claimed. Note that for $r$ as above, Lemma 2.1 tells us that $M$ is faithful, so that Theorem 3.5] is, indeed, applicable.

Remark 3.9. As we pointed out in Remark 3.7] $d_{\mathcal{G}}(\omega(\mathcal{G} / \mathcal{H})) \leq d(\mathcal{G} / \mathcal{H})$. The difference $\operatorname{pr}(\mathcal{G} / \mathcal{H})=d(\mathcal{G} / \mathcal{H})-d_{\mathcal{G}}(\omega(\mathcal{G} / \mathcal{H})) \geq 0$ can be arbitrarily large, even if $\mathcal{H}=\{1\}$. In this case $d(\mathcal{G})=d(\mathcal{G} /\{1\})$ is the minimal number of generators of $\mathcal{G}$, and $\operatorname{pr}(\mathcal{G})=d(\mathcal{G})-d_{\mathcal{G}}(\omega \mathcal{G})$ is usually called the presentation rank or generation gap of $\mathcal{G}$. All solvable groups $\mathcal{G}$ have presentation $\operatorname{rank} \operatorname{pr}(\mathcal{G})=0$; see [Gr, Lectures 6 and 7]. Moreover, if the derived subgroup $[\mathcal{G}, \mathcal{G}]$ is nilpotent then $\operatorname{pr}(\mathcal{G} / \mathcal{H})=0$ holds for every subgroup $\mathcal{H}$ of $\mathcal{G}$; see Ki].

Corollary 3.10. (a) Suppose a group $\mathcal{G}$ of order $n$ can be generated by $r \geq 2$ elements. Then $\tau(A) \leq(r-1) n+1$ for any $\mathcal{G}$-crossed product central simple algebra $A$.

(b) $\tau(A) \leq\left(\left\lfloor\log _{2}(n)\right\rfloor-1\right) n+1$ holds for any crossed product central simple algebra $A$ of degree $n \geq 4$

Here, as usual, $\lfloor x\rfloor$ denotes the largest integer $\leq x$.

Proof. (a) is an immediate consequence Corollary 3.8. (b) follows from (a), because any group of order $n$ can be generated by $r \leq \log _{2}(n)$ elements. (Indeed, $\left|\left\langle\mathcal{G}_{0}, g\right\rangle\right| \geq 2\left|\mathcal{G}_{0}\right|$ for any subgroup $\mathcal{G}_{0}$ of $\mathcal{G}$ and any $g \in \mathcal{G} \backslash \mathcal{G}_{0}$.) Note also that $\left\lfloor\log _{2}(n)\right\rfloor \geq 2$ for any $n \geq 4$.

\section{Proof of Theorem 1.1}

For the next two sections we shall assume that $\mathcal{G}=\mathcal{S}_{n}$ and $\mathcal{H}=\mathcal{S}_{n-1}$. We will use the following standard notations for $\mathcal{S}_{n}$-lattices:

$$
\mathbb{Z}\left[\mathcal{S}_{n} / \mathcal{S}_{n-1}\right]=U_{n} \quad \text { and } \quad \omega\left(\mathcal{S}_{n} / \mathcal{S}_{n-1}\right)=A_{n-1}
$$


The natural generators of $U_{n}$ will be denoted by $u_{1}, \ldots, u_{n}$; the symmetric group $\mathcal{S}_{n}$ permutes them via $\sigma\left(u_{i}\right)=u_{\sigma(i)} . A_{n-1}$ is the sublattice of $U_{n}$ generated by $u_{i}-u_{1}$ as $i$ ranges from 2 to $n$.

Recall that the universal division algebra $\operatorname{UD}(n)$ is generated, as a $k$-division algebra, by a pair of generic $n \times n$-matrices $X$ and $Y$. We may assume without loss of generality that $X$ is diagonal. Following Row $_{2}$ we will denote the diagonal entries of $X$ by $\zeta_{i i}^{\prime}$ and the entries of $Y$ by $\zeta_{i j}$, where $\zeta_{i i}^{\prime}$ and $\zeta_{i j}$ are algebraically independent variables over $k$. The group $\mathcal{S}_{n}$ permutes these variables as follows:

$$
\sigma\left(\zeta_{i i}^{\prime}\right)=\zeta_{\sigma(i) \sigma(i)}^{\prime} \text { and } \sigma\left(\zeta_{i j}\right)=\zeta_{\sigma(i) \sigma(j)}
$$

We identify the multiplicative group generated by $\zeta_{i i}^{\prime}$ with the $\mathcal{S}_{n}$-lattice $U_{n}$ (via $\zeta_{i i}^{\prime} \leftrightarrow u_{i}$ ), and the multiplicative group generated by $\zeta_{i j}$ with $U_{n} \otimes U_{n}$ (via $\left.\zeta_{i j} \leftrightarrow u_{i} \otimes u_{j}\right)$. Consider the exact sequence

$$
0 \rightarrow \operatorname{Ker}(f) \rightarrow U_{n} \oplus U_{n}^{\otimes 2} \stackrel{f}{\rightarrow} A_{n-1} \rightarrow 0
$$

of $\mathcal{S}_{n}$-lattices, where $f\left(u_{i}, u_{j} \otimes u_{h}\right)=u_{j}-u_{h}$. This sequence is the sequence (2.4) of Lemma 2.2 for $\mathcal{G}=\mathcal{S}_{n}$ and $\mathcal{H}=\mathcal{S}_{n-1}$, with two extra copies of $U_{n}$ added: the second copy of $U_{n}$ is the sublattice of $U_{n}^{\otimes 2}$ that is spanned by all elements $u_{i} \otimes u_{i}$. Both copies of $U_{n}$ belong to $\operatorname{Ker}(f)$; in fact,

$$
\operatorname{Ker}(f)=U_{n} \oplus U_{n} \oplus A_{n-1}^{\otimes 2},
$$

where $A_{n-1}^{\otimes 2}$ is identified with the sublattice of $U_{n}^{\otimes 2}$ that is spanned by all elements $\left(u_{i}-u_{j}\right) \otimes\left(u_{l}-u_{m}\right)$.

Let $E=k(\operatorname{Ker}(f))$ and $F=E^{\mathcal{S}_{n}}$. By a theorem of Formanek and Procesi, $F$ is naturally isomorphic to the center $Z(n)$ of $\mathrm{UD}(n)$; see, e.g., $\mathrm{F}_{1}$, Theorem 3]. Note that $E=F\left(\zeta_{11}^{\prime}, \ldots, \zeta_{n n}^{\prime}\right)$ is generated over $F$ by the eigenvalues of the generic matrix $X$. Consequently, $\mathrm{UD}(n)$ is an $\left(E, \mathcal{S}_{n} / \mathcal{S}_{n-1}\right)$-product, and $E^{\mathcal{S}_{n-1}}$ is isomorphic to the maximal subfield $Z(n)(X)$ of $\mathrm{UD}(n)$; see, $[\mathrm{Pr}$, Section II.1].

Theorem 1.1 is now a consequence of the following:

Proposition 4.1. Suppose $n \geq 5$ is odd. Then

(a) $\mathrm{UD}(n)$ is defined over $F_{0}=k\left(\bigwedge^{2} A_{n-1}\right)^{\mathcal{S}_{n}}$,

(b) $Z(n)=k(\operatorname{Ker}(f))^{\mathcal{S}_{n}}$ is rational over $F_{0}=k\left(\bigwedge^{2} A_{n-1}\right)^{\mathcal{S}_{n}}$,

Here, we view $\bigwedge^{2} A_{n-1}$ as the sublattice of antisymmetric tensors in $A_{n-1}^{\otimes 2}$, that is, the $\mathbb{Z}$-span of all $a \wedge a^{\prime}=a \otimes a^{\prime}-a^{\prime} \otimes a$ with $a, a^{\prime} \in A_{n-1}$.

Proof. We will deduce part (a) from Remark 3.4 by constructing a reduced Brauer factor set contained in $E_{0}=k\left(\bigwedge^{2} A_{n-1}\right)$. First we note that the $\mathcal{S}_{n^{-}}$ action on $E_{0}$ is faithful, because $\bigwedge^{2} A_{n-1}$ is a faithful $\mathcal{S}_{n}$-lattice for every $n \geq 4$. (Indeed, $\bigwedge^{2} A_{n-1} \otimes \mathbb{Q}$ is the simple $\mathcal{S}_{n}$-representation corresponding to the partition $\left(n-2,1^{2}\right)$ of $n$; cf. [FH], Exercise 4.6]). 
We now proceed with the construction of the desired Brauer factor set. The computation in $\left[\mathrm{Row}_{2}\right.$, Section 2] shows that the elements

$$
c_{i j h}=\zeta_{i j} \zeta_{j h} \zeta_{i h}^{-1} \in E^{*} .
$$

form a Brauer factor set for $\operatorname{UD}(n)$. By $\operatorname{Row}_{2}$, Theorem 4], if $n$ is odd, $\operatorname{UD}(n)$ has a normalized (and, in particular, reduced) Brauer factor set $\left(c_{i j h}^{\prime}\right)$ given by

$$
c_{i j h}^{\prime}=\left(c_{i j h} / c_{h j i}\right)^{\frac{n+1}{2}}=\left(\zeta_{i j} \zeta_{j i}^{-1} \zeta_{j h} \zeta_{h j}^{-1} \zeta_{h i} \zeta_{i h}^{-1}\right)^{\frac{n+1}{2}} .
$$

Now observe that $\zeta_{i j} \zeta_{j i}^{-1} \zeta_{j h} \zeta_{h j}^{-1} \zeta_{h i} \zeta_{i h}^{-1}$ is precisely the element of $U_{n}^{\otimes 2}$ we identified with $\left(u_{i}-u_{j}\right) \wedge\left(u_{j}-u_{h}\right)$. Thus every $c_{i j h}^{\prime}$ lies in $\bigwedge^{2} A_{n-1} \subset E_{0}$, as desired.

(b) The canonical exact sequence

$$
0 \rightarrow \bigwedge^{2} A_{n-1} \longrightarrow A_{n-1}^{\otimes 2} \longrightarrow \text { Sym }^{2} A_{n-1} \rightarrow 0
$$

of $\mathcal{S}_{n}$-lattices gives rise to an exact sequence

$$
0 \rightarrow \bigwedge^{2} A_{n-1} \rightarrow A_{n-1}^{\otimes 2} \oplus U_{n} \oplus \mathbb{Z} \rightarrow Q \rightarrow 0
$$

where we have put $Q=\operatorname{Sym}^{2} A_{n-1} \oplus U_{n} \oplus \mathbb{Z}$. The crucial fact here is that, by [LL, Section 3.5], if $n$ is odd, $Q$ is a permutation lattice. Applying Proposition 2.4 to the extension of (faithful) $\mathcal{S}_{n}$-fields $E_{0}=k\left(\bigwedge^{2} A_{n-1}\right) \subseteq k\left(A_{n-1}^{\otimes 2} \oplus U_{n} \oplus \mathbb{Z}\right) \cong$ $\left(E_{0}\right)_{\gamma}(Q)$, where $\gamma$ is the image of the class of the above extension in $\operatorname{Ext}_{\mathcal{G}}\left(Q, E_{0}^{*}\right)$, we conclude that

$$
k\left(A_{n-1}^{\otimes 2} \oplus U_{n} \oplus \mathbb{Z}\right) \simeq k\left(\bigwedge^{2} A_{n-1}\right)\left(x_{1}, \ldots, x_{m}\right)
$$

as $\mathcal{S}_{n}$-fields, where $m=\frac{n(n+1)}{2}+1$ and $\mathcal{S}_{n}$ acts trivially on the $x_{i}$ 's. Similarly, putting $L_{n}=A_{n-1}^{\otimes 2} \oplus U_{n}^{2}$, the obvious sequence $0 \rightarrow A_{n-1}^{\otimes 2} \oplus U_{n} \rightarrow L_{n} \rightarrow U_{n} \rightarrow 0$ leads to $k\left(L_{n}\right) \simeq k\left(A_{n-1}^{\otimes 2} \oplus U_{n}\right)\left(t_{1}, \ldots, t_{n}\right)$ as $\mathcal{S}_{n}$-fields. Therefore,

$$
\begin{aligned}
k\left(L_{n}\right) & \simeq k\left(A_{n-1}^{\otimes 2} \oplus U_{n}\right)\left(t_{1}, \ldots, t_{n}\right) \\
& =k\left(A_{n-1}^{\otimes 2} \oplus U_{n} \oplus \mathbb{Z}\right)\left(t_{1}, \ldots, t_{n-1}\right) \\
& \simeq k\left(\bigwedge^{2} A_{n-1}\right)\left(x_{1}, \ldots, x_{m}, t_{1}, \ldots, t_{n-1}\right)
\end{aligned}
$$

as $\mathcal{S}_{n}$-fields, which implies that

$$
Z(n) \simeq k\left(L_{n}\right)^{\mathcal{S}_{n}} \simeq k\left(\bigwedge^{2} A_{n-1}\right)^{\mathcal{S}_{n}}\left(x_{1}, \ldots, x_{m}, t_{1}, \ldots, t_{n-1}\right) ;
$$

so $Z(n)$ is rational over $F_{0}=k\left(\bigwedge^{2} A_{n-1}\right)^{\mathcal{S}_{n}}$.

\section{Proof of Theorem 1.2}

\subsection{Proof of part (a).}

Reduction 5.1. Suppose an algebra $A_{0}$ of degree $n$ has the rational specialization property (see Section 2.4). If Theorem 1.2(a) holds for $A_{0}$ then it holds for any central simple algebra $A$ of degree $n$. 
Proof. Suppose the for some $r \geq 1, A_{0}\left(t_{1}, \ldots, t_{r}\right)$ is defined over a rational extension $F_{0}$ of $k$. Let $A / F$ be an arbitrary central simple algebra of degree $n$. Then by the rational specialization property, $A_{0}$ embeds in $A\left(t_{r+1}, \ldots, t_{s}\right)$ for some $s \gg 0$; thus $A_{0}\left(t_{1}, \ldots, t_{r}\right)$ embeds in $A\left(t_{1}, \ldots, t_{s}\right)$. This shows that $A\left(t_{1}, \ldots, t_{s}\right)$ is defined over $F_{0}$, as desired.

In particular, in proving Theorem 1.2(a), we may assume that $A$ is a division algebra of degree $n$; see Example 2.6, By primary decomposition (cf., e.g., Pi, p. 261]), we only need to consider the cases where $n=2, n=4$ and $n$ is an odd prime. This follows from the next reduction:

Reduction 5.2. If the conclusion of Theorem 1.2(a) holds for central simple algebras $A_{1} / F$ and $A_{2} / F$ (for every choice of the base field $k \subset F$ ) then it also holds for $A=A_{1} \otimes_{F} A_{2}$.

Proof. After replacing $A_{1}$ and $A_{2}$ by, respectively, $A_{1}\left(t_{1}, \ldots, t_{s}\right)$ and $A_{2}\left(t_{1}, \ldots, t_{s}\right)$, we may assume that $A_{1}$ is defined over a subfield $F_{1} \subset F$ such that $k \subset F_{1}$ and $F_{1}$ is rational over $k$. We will now think of $F_{1}$ (rather than $k$ ) as our new base field. After adding more indeterminates, we may assume that $A_{2}$ is defined over a subfield $F_{2} \subset F$, where $F_{1} \subset F_{2}$ and $F_{2}$ is rational over $F_{1}$. Now $F_{2}$ is rational over $k$, and since $A_{1}$ and $A_{2}$ are both defined over $F_{2}$, so is $A$.

We are now ready to complete the proof of Theorem 1.2 (a).

First, suppose $n=2$ or 4 . Since $\operatorname{UD}(n)$ has the rational specialization property, we may assume $A=\mathrm{UD}(n)$; see Reduction 5.1. But since the center of $\mathrm{UD}(n)$ is known to be rational for $n=2$ (see $[\mathrm{Pr}$, Theorem 2.2]) and $n=4$ (see $\left[\mathrm{F}_{2}\right.$ ), these algebras clearly satisfy the conclusion of Theorem 1.2(a). This completes the proof of the theorem for $n=2$ and 4 . We remark that the same argument goes through for $n=3$ (because the center of $\operatorname{UD}(3)$ is known to be rational; see $\left[\mathrm{F}_{1}\right.$ ) and for $n=5,7$ (because the centers of $\operatorname{UD}(5)$ and $\operatorname{UD}(7)$ are known to be stably rational; see $[\mathrm{BL}]$ ), but we shall not need it in these cases.

From now on we will assume that $n=p$ is an odd prime. Then the $\mathcal{S}_{n}$-lattice $A_{n-1}^{\otimes 2}$ is faithful; see Lemma 2.2. Furthermore, by a theorem of Bessenrodt and LeBruyn [BL, Proposition 3] (see also [Be, Lemma 2.8] for a more explicit form of this result), $A_{n-1}^{\otimes 2}$ is permutation projective, i.e., there exists an $\mathcal{S}_{n}$-lattice $L$ such that $P=A_{n-1}^{\otimes 2} \oplus L$ is permutation. We can assume that $k(P)^{\mathcal{S}_{n}}$ is rational over $k$. Indeed, after adding a copy of $U_{n}$ if necessary, we have $P=U_{n} \oplus Q$ for some permutation lattice $Q$, and so $k(P) \simeq k\left(U_{n}\right)(Q)$. Proposition 2.4 implies that $k(P)^{\mathcal{S}_{n}}$ is rational over $k\left(U_{n}\right)^{\mathcal{S}_{n}}$, which in turn is rational over $k$.

Let

$$
i: A_{n-1}^{\otimes 2} \hookrightarrow k\left(A_{n-1}^{\otimes 2}\right)^{*}
$$

and

$$
j: A_{n-1}^{\otimes 2} \hookrightarrow k\left(A_{n-1}^{\otimes 2} \oplus P\right)^{*}
$$


be the natural embeddings of $\mathcal{S}_{n}$-modules. (Here, $j$ identifies $A_{n-1}^{\otimes 2}$ with the first summand of $A_{n-1}^{\otimes 2} \oplus P$.) Recall that by Lemma 3.2 these embeddings, in combination with the exact sequence (2.4) (for $\mathcal{G}=\mathcal{S}_{n}$ and $\mathcal{H}=\mathcal{S}_{n-1}$; see (4.1)), give rise to central simple algebras $\operatorname{Alg}(i)$ and $\operatorname{Alg}(j)$.

By Theorem 3.5. $\mathrm{Alg}(i)$ has the rational specialization property in the class of $\mathcal{S}_{n} / \mathcal{S}_{n-1}$-crossed products. Thus, the universal division algebra $\operatorname{UD}(n)$, being an $\mathcal{S}_{n} / \mathcal{S}_{n-1}$-crossed product (see Example 3.1), is a rational specialization of $\operatorname{Alg}(i)$. Since $\operatorname{UD}(n)$ has the rational specialization property in the class of all central simple algebras of degree $n$ (see Example 2.6), so does $\operatorname{Alg}(i)$.

We claim that $\operatorname{Alg}(j)$ also has the rational specialization property in the class of central simple algebras of degree $n$. Indeed, by Lemma 3.2,

$$
\operatorname{Alg}(j)=\operatorname{Alg}(i) \otimes_{F} \mathcal{S}_{n} E^{\mathcal{S}_{n}},
$$

where $F=k\left(A_{n-1}^{\otimes 2}\right)$ and $E=k\left(A_{n-1}^{\otimes 2} \oplus P\right)$. Now Proposition 2.4 tells us that $E^{\mathcal{S}_{n}}$ is a rational extension of $F^{\mathcal{S}_{n}}$, and the claim follows.

By Reduction 5.1 it now suffices to prove that $\operatorname{Alg}(j)$ is defined over a purely transcendental extension of $k$. Put $E_{0}=k\left(A_{n-1}^{\otimes 2} \oplus(0) \oplus L\right) \subseteq E$. Since the image of $j$ is contained in $E_{0}^{*}$, Lemma 3.3 tells us that $\operatorname{Alg}(j)$ is defined over $E_{0}^{\mathcal{S}_{n}}$. But $E_{0} \simeq k(P)$ and so $E_{0}^{\mathcal{S}_{n}} \simeq k(P)^{\mathcal{S}_{n}}$ which is indeed rational over $k$. This completes the proof of Theorem 1.2 (a).

5.2. Proof of part(b). By the Merkurjev-Suslin Theorem,

$$
\mathrm{M}_{r}(A)=\left(a_{1}, b_{1}\right)_{n_{1}} \otimes_{F} \cdots \otimes_{F}\left(a_{l}, b_{l}\right)_{n_{l}},
$$

for some $r, l \geq 1$, where $(a, b)_{n}$ denotes a symbol algebra; see (1.4).

Let $\lambda_{1}, \ldots, \lambda_{l}, \mu_{1}, \ldots, \mu_{l}$ be $2 l$ central variables, algebraically independent over $F$. We will write $\lambda$ in place of $\left(\lambda_{1}, \ldots, \lambda_{l}\right)$ and $\mu$ in place of $\left(\mu_{1}, \ldots, \mu_{l}\right)$. Then

$$
\begin{array}{r}
\mathrm{M}_{r}(A)(\lambda, \mu)=\left(a_{1}, b_{1}\right)_{n_{1}} \otimes_{K(\lambda, \mu)} \cdots \otimes_{K(\lambda, \mu)}\left(a_{l}, b_{l}\right)_{n_{l}}= \\
\left(a_{1}^{\prime}, b_{1}^{\prime}\right)_{n_{1}} \otimes_{K(\lambda, \mu)} \cdots \otimes_{K(\lambda, \mu)}\left(a_{l}^{\prime}, b_{l}^{\prime}\right)_{n_{l}}= \\
\left(\left(a_{1}^{\prime}, b_{1}^{\prime}\right)_{n_{1}} \otimes_{F_{0}} \cdots \otimes_{F_{0}}\left(a_{l}^{\prime}, b_{l}^{\prime}\right)_{n_{l}}\right) \otimes_{F_{0}} K(\lambda, \mu),
\end{array}
$$

where $a_{i}^{\prime}=a_{i} \lambda_{i}^{n_{i}}$ and $b_{i}^{\prime}=b_{i} \mu_{i}^{n_{i}}$ for $i=1, \ldots, l$ and $F_{0}=k\left(a_{1}^{\prime}, b_{1}^{\prime}, \ldots, a_{l}^{\prime}, b_{l}^{\prime}\right)$. This shows that $\mathrm{M}_{r}(D)(\lambda, \mu)$ is defined over $F_{0}$. It remains to prove that $F_{0}$ is rational over $k$. The $2 l$ elements $a_{1}^{\prime}, b_{1}^{\prime}, \ldots, a_{l}^{\prime}, b_{l}^{\prime}$ are clearly algebraically independent over $F$. Hence, they are algebraically independent over $k$, and consequently, $F_{0}$ is rational over $k$, as claimed.

Remark 5.3. Our proof of Theorem 1.2 can be used to deduce explicit lower bounds on $s$ in parts (a) and (b) from explicit lower bounds in Theorems of Bessenrodt-LeBruyn [BL, Proposition 3] (on $\operatorname{rank}(L)$ ) and Merkurjev-Suslin (on $r$ ). The lowest possible value of $r$ in part (b), called the Merkurjev-Suslin number, is of independent interest; see $\mathrm{Row}_{3}$, Section 7.2]. 


\section{Proof of Theorem 1.3}

Reduction 6.1. In the course of proving Theorem 1.3, we may assume without loss of generality that $A$ is a division algebra.

Indeed, let $D=\operatorname{Alg}(\mu)$, as in Theorem 3.5, with $\mathcal{G}=\mathbb{Z} / m \times \mathbb{Z} / 2$, and $\mathcal{H}=\{1\}$. Then $D$ is a division algebra (see Remark [3.6), and any other $\mathcal{G}$-crossed product $A / F$ is a rational specialization of $D$. Thus, if we know that Theorem 1.3 holds for $D$ then it holds for $A\left(t_{1}, \ldots, t_{s}\right)$, where $t_{1}, \ldots, t_{s}$ are independent variables over $F$. Using induction on $s$, we see that Reduction 6.1 is now a consequence of the following lemma (applied to $B=\mathrm{M}_{m}(A)$, with $r=2, m_{1}=m$ and $\left.m_{2}=2 m\right)$ :

Lemma 6.2. Let $B / K$ be a central simple algebra of degree $d=m_{1} \ldots m_{r}$ and let $t$ be an independent variable over $K$. Assume $K$ contains a primitive root of unity of degree $\operatorname{lcm}\left(m_{1}, \ldots, m_{r}\right)$. If

$$
B(t)=\left(a_{1}(t), b_{1}(t)\right)_{m_{1}} \otimes \cdots \otimes\left(a_{r}(t), b_{r}(t)\right)_{m_{r}}
$$

for some $a_{1}(t), b_{1}(t), \ldots, a_{r}(t), b_{r}(t) \in K(t)$ then

$$
B=\left(a_{1}^{\prime}, b_{1}^{\prime}\right)_{m_{1}} \otimes \cdots \otimes\left(a_{r}^{\prime}, b_{r}^{\prime}\right)_{m_{r}}
$$

for some $a_{1}^{\prime}, b_{1}^{\prime}, \ldots, a_{r}^{\prime}, b_{r}^{\prime} \in K$.

Our proof is based on a standard specialization argument; for the sake of completeness, we supply the details below.

Proof. We may assume that $K$ is an infinite field; otherwise $B$ is a matrix algebra over $K$, and we can take, e.g., $a_{i}^{\prime}=1, b_{i}^{\prime}=-1$ for every $i$.

Choose generators $x_{i}(t)$ and $y_{i}(t)$ for the cyclic subalgebra $\left(a_{i}(t), b_{i}(t)\right)_{m_{i}}$ of $B(t)=B \otimes_{K} K(t)$ such that $x_{i}(t)^{m_{i}}=a_{i}(t), y_{i}(t)^{m_{i}}=b_{i}(t)$, and $x_{i}(t) y_{i}(t)=$ $\zeta_{m_{i}} y_{i}(t) x_{i}(t)$, where $\zeta_{m_{i}}$ is a primitive root of unity of degree $m_{i}$ in $K$. Choose a $K$-basis $b_{1}, \ldots, b_{d^{2}}$ of $B$ and write

$$
x_{i}(t)=\sum_{j=1}^{d^{2}} \alpha_{i j}(t) b_{i} \quad \text { and } \quad y_{i}(t)=\sum_{j=1}^{d^{2}} \beta_{i j}(t) b_{i},
$$

for some $\alpha_{i j}(t), \beta_{i j}(t) \in K(t)$. Since $K$ is an infinite field, we can choose $t_{0} \in K$ such that $\alpha_{i j}\left(t_{0}\right)$ and $\beta_{i j}\left(t_{0}\right)$ are well-defined and

$$
x_{i}\left(t_{0}\right)=\sum_{j=1}^{d^{2}} \alpha_{i j}\left(t_{0}\right) b_{i} \text { and } y_{i}\left(t_{0}\right)=\sum_{j=1}^{d^{2}} \beta_{i j}\left(t_{0}\right) b_{i}
$$

are non-zero. Let $B_{i}$ denote the subalgebra of $B$ that is generated by $x_{i}\left(t_{0}\right)$ and $y_{i}\left(t_{0}\right)$. Then $B_{i}=\left(a_{i}^{\prime}, b_{i}^{\prime}\right)_{m_{i}}$, where $a_{i}^{\prime}=x_{i}\left(t_{0}\right)^{m_{i}}=a_{i}\left(t_{0}\right)$ and $b_{i}^{\prime}=y_{i}\left(t_{0}\right)^{m_{i}}=$ $b_{i}\left(t_{0}\right)$, and $B_{1}, \ldots, B_{r}$ are commuting subalgebras of $B$ of degrees $m_{1}, \ldots, m_{r}$. 
Hence, by the double centralizer theorem (cf., e.g., [Pi, Theorem 12.7]), $B=B_{1} \otimes$ $\cdots \otimes B_{r}$. This completes the proof of Lemma 6.2 and thus of Reduction 6.1.

We now continue with the proof of Theorem 1.3. In the course of the proof we shall use the following notations. Write $\mathcal{G}=\mathbb{Z} / m \times \mathbb{Z} / 2=\left\langle\sigma_{1}, \sigma_{2}\right\rangle$, where $\sigma_{1}^{m}=\sigma_{2}^{2}=1$. Let $K=F\left(\alpha_{1}, \alpha_{2}\right)$, be a maximal $\mathcal{G}$-Galois subfield of $A$, where $\alpha_{1}^{m}=a_{1}$ and $\alpha_{2}^{2}=a_{2}$ are elements of $F$, and

$$
\begin{array}{ll}
\sigma_{1}\left(\alpha_{1}\right)=\zeta_{m} \alpha_{1}, & \sigma_{1}\left(\alpha_{2}\right)=\alpha_{2}, \\
\sigma_{2}\left(\alpha_{1}\right)=\alpha_{1}, & \sigma_{2}\left(\alpha_{2}\right)=-\alpha_{2} .
\end{array}
$$

Here $\zeta_{m} \in F$ is a primitive $m^{\text {th }}$ root of unity, so that $K$ is, indeed, a $\mathcal{G}$-Galois extension of $F$. Note that the statement of Theorem 1.3 assumes that $F$ contains not only a primitive $m$ th root of unity $\zeta_{m}$ but also a primitive $2 m^{\text {th }}$ root of unity $\zeta_{2 m}$; we shall make use of $\zeta_{2 m}$ later in the proof.

By the Skolem-Noether Theorem, there exist units $z_{1}, z_{2} \in A$ such that $z_{i} x z_{i}^{-1}=\sigma_{i}(x)$ for every $x \in K(i=1,2)$. Set

$$
z_{1}^{m}=b_{1} \in F\left(\alpha_{2}\right)^{*}, z_{2}^{2}=b_{2} \in F\left(\alpha_{1}\right)^{*}, \text { and } u=z_{1} z_{2} z_{1}^{-1} z_{2}^{-1} \in K^{*} .
$$

By [AS, Theorem 1.3], the algebra structure of $A$ can be recovered from the $\mathcal{G}$-field $K$ and the elements $u \in K^{*}, b_{1} \in F\left(\alpha_{2}\right)^{*}$ and $b_{2} \in F\left(\alpha_{1}\right)^{*}$. (These elements have to satisfy certain compatibility conditions; the exact form of these conditions shall not concern us in the sequel.) We will write $A=\left(K, \mathcal{G}, u, b_{1}, b_{2}\right)$.

Lemma 6.3. Let $A=\left(K, \mathcal{G}, u, b_{1}, b_{2}\right)$ and $A^{\prime}=\left(K, \mathcal{G}, u^{\prime}, b_{1}^{\prime}, b_{2}^{\prime}\right)$ be $\mathcal{G}$-crossed products. Then $A \otimes_{F} A^{\prime}$ is Brauer equivalent to $\left(K, \mathcal{G}, u u^{\prime}, b_{1} b_{1}^{\prime}, b_{2} b_{2}^{\prime}\right)$.

Proof. The class of $A=\left(K, \mathcal{G}, u, b_{1}, b_{2}\right)$ in the relative Brauer group $\mathbf{B}(K / F)=$ $H^{2}\left(\mathcal{G}, K^{*}\right)$ is given by a normalized 2-cocycle $a: \mathcal{G} \times \mathcal{G} \rightarrow K^{*}$ so that

$$
b_{i}=a\left(\sigma_{i}, \sigma_{i}\right) a\left(\sigma_{i}^{2}, \sigma_{i}\right) \ldots a\left(\sigma_{i}^{m_{i}-1}, \sigma_{i}\right)
$$

holds for $i=1,2$, where $m_{1}=m$ and $m_{2}=2$, and

$$
u=a\left(\sigma_{1}, \sigma_{2}\right) a\left(\sigma_{s}, \sigma_{1}\right)^{-1} \text {. }
$$

Similarly, the class of $A^{\prime}$ is given by a 2-cocycle $a^{\prime}$. Then the class of $A \otimes_{F} A^{\prime}$ is given by the cocycle $a a^{\prime}$; see, e.g., [Pi, Proposition 14.3]. This proves the lemma.

The following alternative ring-theoretic argument was suggested by the referee: Choose $z_{1}, z_{2} \in A$, as in (6.3), and similarly for $z_{1}^{\prime}, z_{2}^{\prime}$ in $A^{\prime}$. The subalgebra $S$ of $A \otimes_{F} A^{\prime}$ generated by $K \otimes 1, z_{1} \otimes z_{1}^{\prime}$ and $z_{2} \otimes z_{2}^{\prime}$, is clearly isomorphic to $\left(K, \mathcal{G}, u u^{\prime}, b_{1} b_{1}^{\prime}, b_{2} b_{2}^{\prime}\right)$. Its centralizer $C_{A \otimes A^{\prime}}(S)$ is an $F$-central simple algebra of degree $2 m=[K: F]$, containing $(K \otimes K)^{\mathcal{G}}$, where $\mathcal{G}$ acts diagonally on $K \otimes K$. Since $\mathcal{G}$ is abelian, $(K \otimes K)^{\mathcal{G}} \simeq F \oplus \cdots \oplus F$, as an $F[\mathcal{G}]$-algebra. In particular, $C_{A \otimes A^{\prime}}$ contains the idempotents of $K \otimes K$ and, hence, is split over $F$. We thus conclude that

$$
A \otimes_{F} A^{\prime} \simeq S \otimes_{F} C_{A \otimes_{F} A^{\prime}}(S) \sim S \simeq\left(K, \mathcal{G}, u u^{\prime}, b_{1} b_{1}^{\prime}, b_{2} b_{2}^{\prime}\right)
$$


as claimed. (Here $\sim$ denotes Brauer equivalence over $F$.)

We now proceed with the proof of Theorem 1.3, using the notations of (6.2) and (6.3). Since $b_{1}=z_{1}^{m} \in K^{\sigma_{1}}=F\left(\alpha_{2}\right)$, we can write

$$
b_{1}=f_{1}+f_{2} \alpha_{2}
$$

for some $f_{1}, f_{2} \in F$.

Lemma 6.4. (a) If $f_{1}=0$ then $A$ is cyclic.

(b) If $f_{2}=0$ then $A=(a, b)_{m} \otimes(c, d)_{2}$, for some $a, b, c, d \in F^{*}$.

Proof. (a) If $f_{1}=0$ then $z_{1}^{2 m}=b_{1}^{2}=f_{2}^{2} a_{2} \in F^{*}$ but $z_{1}^{m}=f_{2} \alpha_{2} \notin F$. Since $F$ contains a primitive root of unity of degree $2 m, F\left(z_{1}\right)$ is a cyclic maximal subfield of $A$ of degree $2 m$; cf. [Lang, Theorem VIII.6.10(b)]. Thus $A$ is a cyclic algebra, as claimed.

(b) If $f_{2}=0$, i.e., $b_{1} \in F$, then the $F$-subalgebra $A_{0}$ of $A$ generated by $z_{1}$ and $\alpha_{1}$ is cyclic of degree $m$. By the double centralizer theorem, $A=A_{m} \otimes Q$, where $Q$ is a quaternion algebra, as claimed.

We are now ready to finish the proof of Theorem 1.3. Lemma 6.4 tells us that Theorem 1.3 is immediate if $f_{1}=0$ or $f_{2}=0$. Thus from now on we shall assume $f_{1} f_{2} \neq 0$.

Now let $A=\left(K, \mathcal{G}, u, b_{1}, b_{2}\right)$ and, for any $f \in F^{*}$, define $A_{f}=\left(K, \mathcal{G}, u, f b_{1}, b_{2}\right)$. Since $\left(a_{1}, f\right)_{m} \otimes_{F} \mathrm{M}_{2}(F) \simeq(K, \mathcal{G}, 1, f, 1)$, Lemma6.3 tells us that $A_{f} \sim\left(a_{1}, f\right)_{m} \otimes_{F}$ $A$, where $\sim$ denotes Brauer equivalence. In other words, $A \sim\left(f, a_{1}\right)_{m} \otimes_{F} A_{f}$. Thus it is enough to show that $A_{f}$ is cyclic, for some $f \in F^{*}$.

To prove the last assertion, observe that if we expand $\left(z_{1}+\alpha_{1}\right)^{m}$ then all terms, other than $z_{1}^{m}$ and $\alpha_{1}^{m}$, will cancel. (For a simple proof of this fact, due to Bergman, see Row $_{3}$, p. 195]). Thus, if $\gamma=z_{1}+\alpha_{1}$ then

$$
\gamma^{m}=z_{1}^{m}+\alpha_{1}^{m}=f b_{1}+a_{1}
$$

in $A_{f}$. Setting $f=-\frac{a_{1}}{f_{1}} \in F^{*}$, we obtain $\gamma^{m}=c \alpha_{2}$, where $c=-\frac{a_{1} f_{2}}{f_{1}} \in F^{*}$; see (6.4). Thus $\gamma^{2 m}=c^{2} a_{2} \in F^{*}$ but $\gamma^{m} \notin F$. Since $F$ contains a primitive $2 m^{\text {th }}$ root of unity, $F(\gamma) / F$ is a cyclic field extension of degree $2 m$. In other words, $F(\gamma)$ a cyclic maximal subfield of $A_{f}$, and $A_{f}$ is a cyclic algebra of degree $2 m$, as claimed.

\section{THE FIELD OF DEFINITION OF A QUADRATIC FORM}

7.1. Preliminaries. Let $V=F^{n}$ be an $F$-vector space, equipped with a quadratic form $q: V \rightarrow F$. Recall that $q$ is said to be defined over a subfield $F_{0}$ of $F$ if $q=q_{F_{0}} \otimes F$, where $q_{F_{0}}$ is a quadratic form on $V_{0}=F_{0}^{n}$. Is easy to see that $q$ is defined over $F_{0}$ if and only if $V$ has an $F$-basis $e_{1}, \ldots, e_{n}$ such that $b\left(e_{i}, e_{j}\right) \in F_{0}$, where $b: V \times V \rightarrow F$ is the symmetric bilinear form associated to $q$ (i.e., $q(v)=b(v, v))$. 
We shall always assume that $\operatorname{char}(F) \neq 2$ and $F$ (and $F_{0}$ ) contain a base subfield $k$. As usual, we shall write $<a_{1}, \ldots, a_{n}>$ for the diagonal form

$$
a_{n} x_{1}^{2}+\cdots+a_{n} x_{n}^{2}
$$

and $\ll a_{1}, \ldots, a_{n} \gg$ for the Pfister form $<1, a_{1}>\otimes \cdots \otimes<1, a_{n}>$. Given a quadratic form $q$ we shall ask:

(a) What is the smallest value of $\operatorname{trdeg}_{k}\left(F_{0}\right)$, where $q$ is defined over $F_{0}$ ? We shall denote this number by $\tau(q)$.

(b) Can $q$ be defined over a rational extension $F_{0}$ of $k$ ?

These are the same questions we asked for central simple algebras in the Introduction. In the case of quadratic forms our answers are more complete (and the proofs are easier).

Proposition 7.1. Let $V=F^{n}$ and let $q: V \rightarrow F$ be a quadratic form on $V$. Then

(a) $\tau(q) \leq n$. Moreover, if $a_{1}, \ldots, a_{n}$ are independent variables over $k, F=$ $k\left(a_{1}, \ldots, a_{n}\right)$, and $q=<a_{1}, \ldots, a_{n}>$ then $\tau(q)=n$.

(b) Let $t_{1}, \ldots, t_{n}$ be independent variables over $F$. Then $q^{\prime}=q \otimes_{F} F\left(t_{1}, \ldots, t_{n}\right)$ is defined over a rational extension $F_{0}$ of $k$.

Proof. Diagonalizing $q$, write $q=<a_{1}, \ldots, a_{n}>$ in the basis $e_{1}, \ldots, e_{n}$.

(a) To prove the first assertion, set $F_{0}=k\left(a_{1}, \ldots, a_{n}\right)$. Then $q$ is defined over $F_{0}=k\left(a_{1}, \ldots, a_{n}\right)$ and $\operatorname{trdeg}_{k}\left(F_{0}\right) \leq n$, as desired. For the proof of the second assertion see $\left[\mathrm{Re}_{2}\right.$, Proof of Theorem 10.3].

(b) Set $a_{i}^{\prime}=t_{i}^{2} a_{i}$. Then $q^{\prime}=<a_{1}, \ldots, a_{n}>=<a_{1}^{\prime}, \ldots, a_{n}^{\prime}>$ over $F\left(t_{1}, \ldots, t_{n}\right)$. Hence, $q^{\prime}$ is defined over $F_{0}=k\left(a_{1}^{\prime}, \ldots, a_{n}^{\prime}\right)$. We claim that $F_{0}$ is rational over $k$. Indeed, since the nonzero elements of $\left\{a_{1}^{\prime}, \ldots, a_{n}^{\prime}\right\}$ are algebraically independent over $F$, they are algebraically independent over $k$, and the claim follows.

In the sequel we shall need the following analogue of Lemma 2.7.

Lemma 7.2. Let $q$ be a quadratic form defined over $F, t_{1}, \ldots, t_{r}$ be independent variables over $F$, and $F^{\prime}=F\left(t_{1}, \ldots, t_{r}\right)$. Set $q^{\prime}=q \otimes_{F} F^{\prime}$. Then $\tau(q)=\tau\left(q^{\prime}\right)$.

Proof. The inequality $\tau\left(q^{\prime}\right) \leq \tau(q)$ is obvious from the definition of $\tau(q)$. To prove the opposite inequality, we may assume $F$ is an infinite field; otherwise $\tau(q)=0$, and there is nothing to prove. We may also assume $r=1$; the general case will then follows by induction on $r$. Let $b^{\prime}$ be the symmetric bilinear form associated to $q^{\prime}$ and choose a basis $b_{1}(t), \ldots, b_{n}(t)$ of $\left(F^{\prime}\right)^{n}$ so that $\operatorname{trdeg}_{k} k\left(\alpha_{i j}(t)\right)=\tau\left(q^{\prime}\right)$, where $\alpha_{i j}(t)=b^{\prime}\left(b_{i}(t), b_{j}(t)\right)$. Since $F$ is an infinite field, we can find a $c \in F$ such that (i) the vectors $b_{1}(c), \ldots, b_{d}(c)$ are welldefined and form a basis of $F^{d}$, and (ii) each $\alpha_{i j}(c)$ is well-defined. Now $q$ is defined over $k\left(\alpha_{i j}(c)\right)$ and thus

$$
\tau(q) \leq \operatorname{trdeg}_{k}\left(\alpha_{i j}(c)\right) \leq \operatorname{trdeg}_{k}\left(\alpha_{i j}(t)\right)=\tau\left(q^{\prime}\right)
$$


as claimed.

7.2. Proof of Theorem 1.5. Let $F$ be a field containing a primitive $4^{\text {th }}$ root of unity. Note that for the purpose of proving Theorem 1.5, we may assume that $A / F$ is a division algebra. Otherwise, $A$ is isomorphic to $\mathrm{M}_{4}(F)$ or to $\mathrm{M}_{2}(D)$, where $D=(a, b)_{2}$ is a quaternion algebra. Thus, $A$ is defined over $k$ or over the field $F_{0}=k(a, b)$, respectively, and so is the trace form of $A$. Alternatively, a simple direct computation shows that the trace form of $\mathrm{M}_{2}(E)$ is trivial (and thus is defined over $k$ ) for any central simple algebra $E / F$.

From now on we will assume that $A / F$ is a division algebra of degree 4 . By a theorem of Albert $[\mathrm{A}, A$ is a $\mathcal{G}$-crossed product, with $\mathcal{G}=\mathbb{Z} / 2 \times \mathbb{Z} / 2$. Let $K$ be a $\mathcal{G}$-Galois maximal subfield. Using the notations introduced in Section 6 (with $m=2$ ), we write $\mathcal{G}=\left\langle\sigma_{1}, \sigma_{2}>, K=F\left(\alpha_{1}, \alpha_{2}\right), \alpha_{i}^{2}=a_{i} \in F\right.$ and $A=\left(K, \mathcal{G}, u, b_{1}, b_{2}\right)$ for some $u \in K^{*}, b_{1} \in F\left(\alpha_{2}\right)=K^{\sigma_{1}}$, and $b_{2} \in F\left(\alpha_{1}\right)=K^{\sigma_{2}}$. Set $\sigma_{3}=\sigma_{1} \sigma_{2} \in \operatorname{Gal}(K / F) ; z_{3}=\left(z_{1} z_{2}\right)^{-1}, \alpha_{3}=\alpha_{1} \alpha_{2}, a_{3}=\alpha_{3}^{2}=a_{1} a_{2}, b_{3}=z_{3}^{2}$ (so that $b_{i}=z_{i}^{2}$ for $i=1,2,3$ ), and

$$
t_{i}=\frac{1}{2} \operatorname{Tr}_{K^{\sigma_{i} / F}}\left(z_{i}^{2}\right), \quad n_{i}=N_{K^{\sigma_{i} / F}}\left(z_{i}^{2}\right)
$$

for $i=1,2,3$.

Our proof of Theorem [1.5 is based on the following result of Serre [Se, $\mathrm{RST}$.

Proposition 7.3. Suppose $z_{1}$ and $z_{2}$ are chosen so that $t_{i} \neq 0$ and $n_{i}^{2}-t_{i} \neq 0$ for any $i=1,2,3$. Then the trace form $q$ of $A$ is Witt-equivalent (over $F$ ) to $q_{2} \oplus q_{4}$, where

$$
q_{2}=\ll n_{1}-t_{1}^{2}, n_{2} \gg
$$

is a 2-fold Pfister form and

$$
q_{4}=\ll t_{1}-n_{1}^{2},\left(n_{2}-t_{2}^{2}\right) n_{2}, t_{1} t_{2}, t_{2} t_{3} \gg
$$

is a 4-fold Pfister form.

We claim that for the purpose of proving Theorem[1.5, we may assume without loss of generality that $t_{i} \neq 0$ and $n_{i}^{2}-t_{i} \neq 0$ for any $i=1,2,3$. Indeed, in view of Lemma 7.2 it suffices to prove Theorem 1.5 for a single division algebra $A$ which has the rational specialization property in the class of algebras of degree 4, e.g., for $A=\mathrm{UD}(4)$; see Remark 2.8. Thus we only need to show that in this algebra $t_{i} \neq 0$ and $n_{i}-t_{i}^{2} \neq 0$ for any choice of $z_{1}, z_{2}$.

Indeed, we may assume without loss of generality that $i=1$ (the cases where $i=2$ and 3 will then follow by symmetry). Write $b_{1}=f_{1}+f_{2} \alpha_{2}$ for some $f_{1}, f_{2} \in F$, where $t_{1}=f_{1}$ and $n_{1}-t_{1}^{2}=f_{2}^{2} a_{2}$. Lemma 6.4 shows that if $t_{1}=0$ then $A$ is cyclic and if $n_{1}-t_{1}^{2}=0$ then $A$ is biquaternion. But since our algebra $A$ has the rational specialization property, it is neither cyclic nor biquaternion. We conclude that $t_{1}\left(n_{1}-t_{1}^{2}\right) \neq 0$, as claimed. 
We now proceed to simplify the form given by Proposition 7.3. After expanding $q_{2}$ and $q_{4}$, cancelling the common term $<1, t_{1}^{2}-n_{1}>$ (which can be done, since we are assuming $\sqrt{-1} \in F$ ) and dividing some of the entries by elements of $\left(F^{*}\right)^{2}$, we see that the trace form of $A$ is Witt equivalent to the 16-dimensional form

$$
q=<1,1-\frac{n_{1}}{t_{1}^{2}}>\otimes\left(<\frac{n_{2}}{t_{2}^{2}}>\oplus \ll\left(1-\frac{n_{2}}{t_{2}^{2}}\right) \frac{n_{2}}{t_{2}^{2}}, t_{1} t_{2}, t_{2} t_{3} \gg_{0}\right),
$$

where $\ll \lambda_{1}, \ldots, \lambda_{r} \gg_{0}$ is defined as a $2^{r}-1$-dimensional form such that $\ll \lambda_{1}, \lambda_{2}, \ldots, \lambda_{r} \gg_{0} \oplus<1>$ is the $n$-fold Pfister form $\ll \lambda_{1}, \lambda_{2}, \ldots, \lambda_{r} \gg$.

Note that since $q$ and the trace form of $A$ are Witt equivalent 16-dimensional forms, the Witt Decomposition Theorem implies that they are, in fact, the same (i.e., isometric). We now observe that all entries of $q$ lie in the subfield $F_{0}=$ $k\left(\frac{n_{1}}{t_{1}^{2}}, \frac{n_{2}}{t_{2}^{2}}, t_{1} t_{2}, t_{2} t_{3}\right)$ of $F$. Thus the trace form of $A$ is defined over $F_{0}$. Since $F_{0}$ is generated by 4 elements over $k$, we have $\operatorname{trdeg}_{k} F_{0} \leq 4$. This completes the proof of Theorem 1.5.

\section{REFERENCES}

[A] A. A. Albert, Structure of Algebras, Amer. Math. Soc. Colloq. Publ. 24, 1939.

[AS] S. A. Amitsur, S. J. Saltman, Generic abelian crossed products and p-algebras, J. Algebra, 51 (1978), 76-87.

[Be] E. Beneish, Induction theorems on the stable rationality of the center of the ring of generic matrices, Trans. Amer. Math. Soc. 350 (1998), 3571-3585.

[BL] C. Bessenrodt and L. Le Bruyn, Stable rationality of certain PGL $L_{n}$-quotients, Invent. Math. 104 (1991), 179-199.

[FSS] B. Fein, D. J. Saltman and M. Schacher, Embedding problems for finite dimensional division algebras, J. Algebra 167 (1994), 588-626.

$\left[\mathrm{F}_{1}\right] \quad$ E. Formanek, The center of the ring of $3 \times 3$ generic matrices, Linear and Multilinear Algebra 7 (1979), 203-212.

$\left[\mathrm{F}_{2}\right] \quad$ E. Formanek, The center of the ring of $4 \times 4$ generic matrices, J. Algebra $62(1980)$, no. 2, 304-319.

[FH] W. Fulton and J. Harris, Representation Theory, A First Course, Springer-Verlag, New York, 1991.

[Gr] K. W. Gruenberg, Relation Modules of Finite Groups, CBMS Regional Conference Series in Mathematics, No. 25, Amer. Math. Soc., Providence, 1976.

[J] N. Jacobson, Basic Algebra II, 2nd ed., W.H. Freeman and Co., New York, 1989.

[Ki] W. Kimmerle, Relative relation modules as generators for integral group rings of finite groups, Math. Z. 172 (1980), 143-156.

[Lam] T.-Y. Lam, The Algebraic Theory of Quadratic Forms, W. A. Benjamin Inc., 1973.

[Lang] S. Lang, Algebra, Addison-Wesley Publishing Co., Inc., 1965.

[Le] N. Lemire, Essential dimension of algebraic groups and integral representations of Weyl groups, preprint.

Available at http://www.math.uwo.ca/nlemire/preprints.html.

[LL] N. Lemire and M. Lorenz, On certain lattices associated with generic division algebras, J. Group Theory 3 (2000), 385-405. 
[Le] H. W. Lenstra, Rational functions invariant under a finite abelian group, Invent. Math. 25 (1974), 299-325.

[Mas] K. Masuda, On a problem of Chevalley, Nagoya Math. J. 8 (1955), 59-63.

[Pa] D. S. Passman, The Algebraic Structure of Group Rings, John Wiley \& Sons, New York, 1977.

[Pi] R. S. Pierce, Associative Algebras, Springer-Verlag, New York, 1982.

[Pr] C. Procesi, Non-commutative affine rings, Atti Acc. Naz. Lincei, S. VIII, v. VIII, fo. 6 (1967), 239-255.

$\left[\mathrm{Re}_{1}\right] \quad$ Z. Reichstein, On a theorem of Hermite and Joubert, Canadian J. Math., 51 (1) (1999), 69-95.

$\left[\mathrm{Re}_{2}\right] \quad$ Z. Reichstein, On the notion of essential dimension for algebraic groups, Transformation Groups, 5, no. 3 (2000), 265-304.

[RV] Z. Reichstein and N. Vonessen, An embedding property of universal division algebras, J. Algebra 177 (1995), 451-462.

[Ro] S. Rosset, Group extensions and division algebras, J. Algebra 53 (1978), 297-303.

[Rost] M. Rost, Computation of some essential dimensions, preprint. Available at http://www.math.ohio-state.edu/rrost/ed.html.

[RST] M. Rost, J.-P. Serre, J.-P. Tignol, The trace form of a central simple algebra of degree four, in preparaton.

[Row ${ }_{1}$ L. H. Rowen, Polynomial Identities in Ring Theory, Academic Press, 1980.

[Row 2 ] L. H. Rowen, Brauer factor sets and simple algebras, Trans. Amer. Math. Soc., 282, no. 2 (1984), 765-772.

[Row 3 ] L. H. Rowen, Ring Theory, vol. 2, Academic Press, 1988.

[Row $\left.{ }_{4}\right]$ L. H. Rowen, Division algebras over $C_{2}$ - and $C_{3}$-fields, Proc. Amer. Math. Soc. 130 (2002), no. 6, 1607-1610

[RS] L. H. Rowen and D. J. Saltman, Normalized Brauer factor sets, J. Algebra 198 (1997), 446-468.

$\left[\mathrm{Sa}_{1}\right] \quad$ D. J. Saltman, Twisted multiplicative field invariants, Noether's problem, and Galois extensions, J. Algebra 131 (1990), 535-558.

$\left[\mathrm{Sa}_{2}\right] \quad$ D. J. Saltman, A note on generic division algebras, Contemp. Math. 130 (1992), 385-394.

$\left[\mathrm{Sa}_{3}\right] \quad$ D. J. Saltman, Lectures on Division Algebras, CBMS Regional Conference Series in Mathematics, No. 94, Amer. Math. Soc., Providence, 1999.

[Se] J.-P. Serre, letters of November 16 and November 24, 1998.

[Sh] I. R. Shafarevich, Basic Algebraic Geometry, vol. 1, second edition, Springer-Verlag, 1994.

[S] R. L. Snider, Is the Brauer group generated by cyclic algebras?, in "Ring Theory. Waterloo 1978", Lect. Notes in Math. 734 (1979), Springer, 279-301. 
Department of Mathematics, Temple University, Philadelphia, PA 19122-6094

E-mail address: lorenz@math.temple.edu

$U R L:$ http://www. math.temple.edu/ lorenz

Department of Mathematics, University of British Columbia, Vancouver, BC, CANADA, V6T $1 Z 2$

E-mail address: reichst@math.ubc.ca

URL: http://www.math.ubc.ca/ $\sim$ reichst

Department of Mathematics and Computer Science, Bar-Ilan University, RAMAT-GAN 52900, ISRAEL

E-mail address: rowen@macs.biu.ac.il

Department of Mathematics, University of Texas, Austin, TX 78712

E-mail address: saltman@math.utexas.edu 